\title{
Recherches faunistiques et écologiques sur les Hydracariens de I'Apennin (Italie). Le genre Torrenticola, Piersig (Acari, Actinedida, Torrenticolidae) ${ }^{2}$
}

\author{
B. Cicolani 1 \\ A. Di Sabatinol
}

Mots clés : Hydracariens, eaux courantes, hydrobiologie, Apennins, Italie.

Ce travail concerne les données faunistiques et écologiques relatives aux especes italiennes du gense Torrenticola tecueillies dans l'Apennin. Treize espèces ont été signalées, cinq sont nouvelles pour la faune italienne (T. similis, T. lativalvata, $T$. brevirostris, $T$. ungeri et $T$. amplexa). L'étude des caractères dimensionels a permis d'individualiser quelques indices morphologiques de valeur discriminante à un niveau spécifique et a permis de mettre en synonymie quatre espèces. Une distribution différente des espèces entre les secteurs de l'Apennin abruzzin et calabrais a été aussi mise en évidence. L'importance du microhabitat, déterminée par la typologie du cours d'eau, a été soulignée afin d'expliquer la distribution géographique des espèces dans le bassin méditerranéen.

Faunistic and ecological research on the water mites of the Apennines (Italy). The Genus Torrenticola Piersig (Acari, Actinedidae, Torrenticolidae).

Keywords: Water mites, running waters, hydrobiology, Apennines, Italy.

Faunistic and ecological data on the distribution of the genus Torrenticola in the Apennines (Italy) are reported. Thirteen species were collected ; the species $T$. similis, $T$. lativalvata, $T$. brevirostris, $T$. ungeri and $T$. amplexa are new to Italian fauna. The study of dimensional characters generated some morphological indices with discriminant values at the species-level, and consequently in the synonymization of four species. A differential distribution of the species between the Abruzzian and Calabrian sectors of the Apennines, was also found. The river typology and the availability of peculiar microhabitats, were assumed to be important factors in explaining the geographical distribution of these species in the Mediterranean basin.

\section{Introduction}

Les hydracariens appartenant à la faune des Apennins n'ont jamais fait l'objet de recherches programmées, et des lacunes en résultent, așsez graves pour nos connaissances faunistique et systématique.

Jusqu'il y a dix ans, avant d'avoir commencé à faire des recherches sur les Hydracariens de l'Apennin (Cicolani \& Sisino 1983, Cicolani \& Di Sabatino $1985 \mathrm{a}, 1985 \mathrm{~b}$ ), on connaissait seulement les données concernant certains fleuves de l'Ombrie et des

1. Dipartimento di Scienze Ambientali, Université de L'Aquila, Via Vetoio 20, 67100 L'Aquila Italie.

2. Contribution VIII à la connaissance des Hydracariens de l'Apennin. Recherche effectuée avec la contribution financière du MPl $40 \%$ et $60 \%$.
Marches (Viets 1955, Maglio 1956). Les études successives ont permis la récolte d'un abondant matériel appartenant à presque 150 espèces, 34 genres et 18 familles.

Dans la présente note sont exposés les résultats relatifs au genre Torrenticola, qui présente beaucoup de difficultés pour la détermination spécifique, déjà soulignées par de nombreux auteurs (Angelier 1954, Lundblad 1942, 1956, Bader 1988).

Actuellement le genre, dont la composition mondiale et européenne est respectivement de quelques 300 et 60 espèces, est représenté en Italie par 8 espèces appartenant aux sous-genres Torrenticola s.5. et Monatractides : $T$. anomala (Koch 1837), $T$. maglioi (Koenike 1908), T. elliptica (Maglio 1909), 
T. mucronata (Viets 1955), T. crenobia (Di Sabatino \& Cicolani 1989), T. meridionalis (Di Sabatino \& Cicolani 1990), $T$. stadleri (Walter 1924) et $T$. madritensis (Viets 1930).

\section{Les stations étudiées, matériel et méthodes}

La zone étudiée (fig. 1) est celle de la dorsale des Apennins et des zones de piémont et des plaines, reliées suivant le critère géographique et non biogéographique. Elle a été subdivișée en Apennin septentrional (Colle di Cadibona - Bocca Serriola), Apennin central (Bocca Serriola - Rionero Sannitico), Apennin méridional (Passo di Rionero Sannitico - Aspromonte) et Apennin sicilien (jusqu'au groupe des Madonie) ultérieurement subdivisés en secteurs ou en districts orohydrographiques ne permettant pas encore une analyse des espèces selon une distribution par bassins hydrographiques.

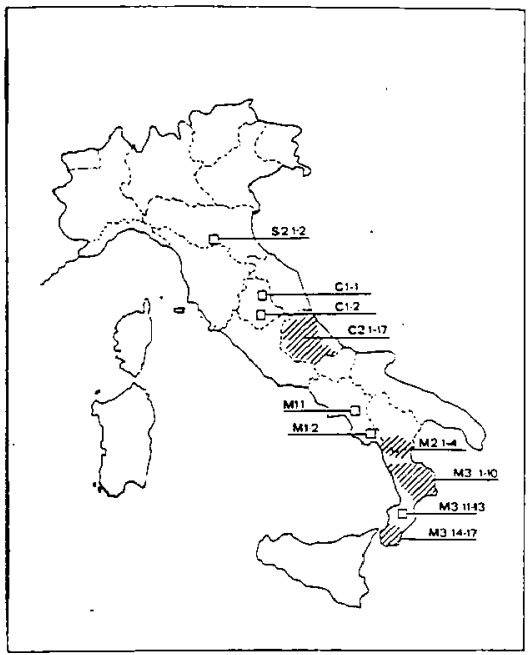

Fig. 1. Localisation des stations. (S2-Ap, toscaneemilien ; C1-Ap, ombrien-des Marches ; C2-Ap, abruzzain ; M1-Ap, campanien ; M2-Ap, lucanien ; M3-Ap, calabrais).
Les stations ont été choisies dans le but de pouvoir représenter les différentes typologies fluviales: ruisseaux, torrents, rivières impétueuses aux forts étiages d'été.

Le tableau I réunit les données relatives aux stations échantillonnées, à la dénomination des cours d'eau, à l'altitude et à la température du l'eau. D'après l'examen de la liste, on a déduit les données relatives à l'Apennin ligurien et sicilien qui ne sont pas encore complètes ; elles feront l'objet de prochaines publications.

Le genre Torrenticola a été récemment subdivisé en 7 sous-genres (Bader 1988) même si la plupart des auteurs ne sont pas d'accord avec cette subdivision. Dans l'attente d'études plus appronfondies sur la validité des caractères actuellement considérés, nous suivons les indications de l'auteur suisse.

L'identification de la plupart des espèces présente de nombreuses difficultés et opportunément Bader (1988) retient comme nécessaire, même si ce n'est pas toujours suffisant, l'examen global de 18 caractères morphologiques sur les faces dorsale et ventrale et dans le gnathosome $\sigma$ et $q$.

Le peu d'acarothèques existant dans les musées et les universités italiennes, le manque de clarté dans les descriptions originales, la présence de grossières erreurs de détermination dans certains travaux d'auteurs, italiens ou non, nous ont conduit à vérifier personnellement les données en demandant du matériel aux plus importants musées européens. Ainsi nous avons pu examiner les collections du Swedish Museum of Natural History de Stockholm, du Forschungsinstitut Senckenberg de Frankfurt, et du Naturhistorisches Museum de Bâle. Ce matériel et celui qui a été recueilli par nous et par nos collègues, a été examiné et utilisé pour effectuer des mesures en vue de comparaisons statistiques entre les populations de différentes provenance. Les 24 caractères considérés (Fig. 2) ont été :

\footnotetext{
1 = Longueur écusson dorsal.

2 = Largeur écusson dorsal.

3 = Longueur face ventrale (y compris la pointe des épimères I).

$4=$ Largeur ventrale.

5 = Longueur plaque dorsale inférieure.

6 = Longueur plaque dorsale supérieure.

7 = Profondeur du capitulum.

8 = Ampleur capitulum.

9 = Longueur épimère $I$ ( $\mathrm{y}$ compris le capitulum).

$10=$ Longueur épimère II et III.
} 

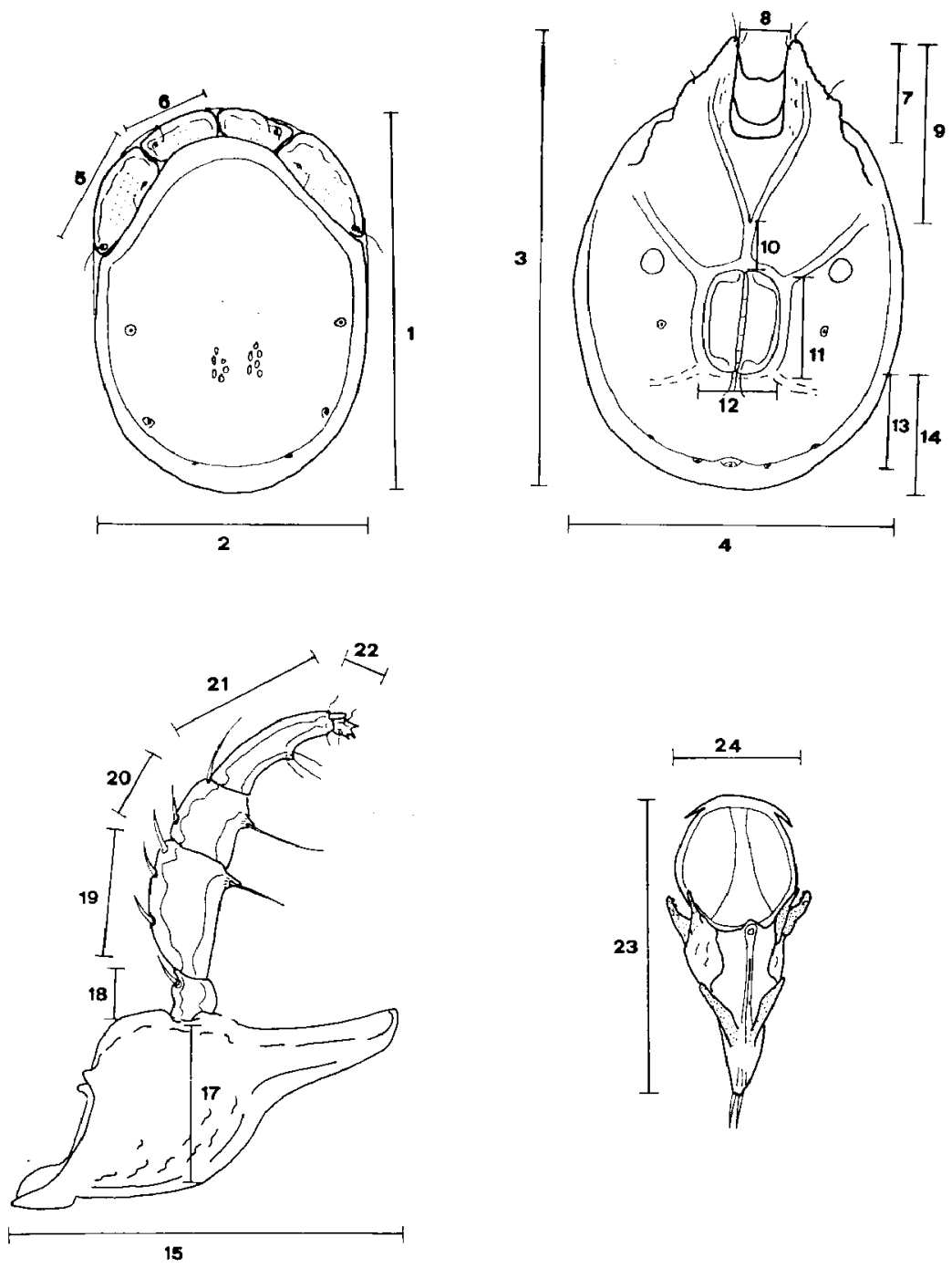

Fig. 2. Représentation des caractères morphologiques (numérotés comme dans le texte). 
Tableau I. Liste des stations prospectées.

( $F=$ Fleuves $; T=$ Torrents $; \mathbf{F} \cdot \mathbf{r a}=$ Fleuves caractérisés par des crues imprévues $;$ Af $=$ Affluents $)$.

\begin{tabular}{|c|c|c|c|c|c|c|c|}
\hline stut. & -ijigu & localiti & prov & 1. date & leg. & nit. & Temp. \\
\hline & & $\begin{array}{l}\text { APENAIM. SEPTENT } \\
\text { 5.2- TOSCAME-EI }\end{array}$ & $\begin{array}{l}\text { RIOANA } \\
\text { IJLIEN }\end{array}$ & & & & \\
\hline $\begin{array}{l}52-1 \\
\$ 2-2\end{array}$ & $\begin{array}{l}\text { T. SCOL TENANA } \\
\text { T.RIORI' }\end{array}$ & $\begin{array}{l}\text { Riolunato (Abetone) } \\
\text { Dardagnd (Abet one) }\end{array}$ & $\begin{array}{l}(00) \\
(B 0)\end{array}$ & $\begin{array}{l}18 / 5 / 84 \\
18 / 5 / 84\end{array}$ & Cicolani & $\begin{array}{l}800 \\
900\end{array}$ & $\overline{-}$ \\
\hline & & $\begin{array}{l}\text { APENHIN LENTRAL } \\
\text { C.1- OHBH]EN-DE }\end{array}$ & S MAR & LCHES & & & \\
\hline Cl-1 & F.PAEL IA & Font1 Tiber 10, Subbissone & [IR] & $23 / 6 / 82$ & Cienficconi & - & _ \\
\hline Cl-2 & F. ANIEME & - C.2- ABPULLLIN & - & - & " & - & - \\
\hline C2-1 & F. WOMANO & Provvidenza & (AD] & $15 / 6 / 84$ & Oi Sabdtino & 1100 & 6.5 \\
\hline$c 2-2$ & F. VGMANO & Ortolana & (AOA) & $15 / 6 / 84$ & 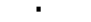 & 1000 & 7.2 \\
\hline $\mathrm{C2}_{2}-3$ & F, WOMANO & Senarica & (TE) & $17 / 6 / 84$ & - & 800 & 8.0 \\
\hline $\mathrm{C} 2-4$ & F. vamalo & Montorio al Vosano & (TE) & $17 / 7 / 85$ & . & 240 & 8.5 \\
\hline$[2-5$ & 8. WOHAMC & Val Voutano & (IE) & 17/7/85 & - & 200 & 11.5 \\
\hline C2-6 & T. S. EIDVAMII & I Pravridenza & (AD) & $15 / 4 / 66$ & - & 1100 & 6.0 \\
\hline$[2-7$ & F. MAVONE & Isala G.Sassa & [IE] & $12 / 6 / 84$ & - & 800 & 9.0 \\
\hline$[2-8$ & F.ATERNO & S.Pelina & IAQ: & $9 / 10 / 85$ & - & 800 & 10.0 \\
\hline$[2-9$ & T. DRTE & Carasanico & (CH) & $12 / 5 / 85$ & Recchis & 500 & - \\
\hline (2-1 & T. ORTE & Bolognano & (CH) & $12 / 5 / 65$ & . & 300 & - \\
\hline$[2-1$ & F. ORFENTO & Rif.delta Cesa & (CH) & $22 / 4 / 66$ & Dell Agatz & 1000 & 6.5 \\
\hline$[2-12$ & F. ORFENTO & Nord da salle & (CH) & $19 / 4 / 85$ &. & 700 & 8.0 \\
\hline$[2-13$ & F. ORFENTO & Sod di Salle & (CH) & 22/3/85 & - & 560 & B.5 \\
\hline$[2-14$ & F. ALEHTO & Roccanontepi ano & (CH) & $15 / 3 / \mathrm{BJ}$ & - & 350 & 9.0 \\
\hline$[2-15$ & F.ALENTO & Bucchianica & (CH) & $12 / 4 / \mathrm{BJ}$ & - & 250 & 12.0 \\
\hline$[2-16$ & F. SANGRO & Barrea & (AO) & $10 / 10 / 87$ & D1 Sabatino & 920 & 6.0 \\
\hline C $2-17$ & F. SAMGRO & Al tedent. & (\$O) & $10 / 10 / \theta 7$ & 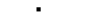 & 880 & 7.0 \\
\hline
\end{tabular}

\begin{tabular}{|c|c|c|c|c|c|c|c|}
\hline \multicolumn{8}{|c|}{$\begin{array}{l}\text { APENMIN MERIDIONAL } \\
\text { M.1 - CAMPANIEN }\end{array}$} \\
\hline$n+1$ & F. SELE & Fiocche & [SA] & $22 / 7 / 85$ & Subugar & 20 & - \\
\hline$n-2$ & F. EUSSENTO & Policastro Bus. & [SA] & $23 / 7 / 85$ & . & 1 & - \\
\hline \multicolumn{8}{|c|}{ M. 2- Lucanien } \\
\hline$n 2-1$ & F. MERCURE & Ponte Mercure & (P1) & $7 / 8 / 86$ & Gerecke & 320 & 19.5 \\
\hline $12-2$ & 1. SAKD ССОSB & P.te Carnuto & (p) & $7 / 9 / 86$ & & $\$ 10$ & 14.8 \\
\hline $\mathrm{H} 2-3$ & RUSCELLO in & Serro del Prete & $(P Z)$ & $8 / 8 / 86$ & - & 1600 & 18.0 \\
\hline $12-4$ & F.LAD & Pte Castelluccio & (PI) 1 & $10 / 6 / 85$ & D) Sabatino & 185 & 17.0 \\
\hline \multicolumn{8}{|c|}{ M.3- CALABRE } \\
\hline A3 -1 & F. CARONTE & Pantanol ungo & (CS) & $24 / 7 / 86$ & Gerecte & 600 & 15.0 \\
\hline H3 -2 & F. CORACE & S.floro & (CI) & $17 / 7 / 85$ & • & 33 & 24.6 \\
\hline กร -3 & F.ALLI & Belladonnz & (Ct) & $17 / 7 / 85$ & - & 0 & 29.4 \\
\hline$n 3-4$ & F. CROCCHIO & Cropani & (cl) & $17 / 7 / 95$ & - & 50 & 15.2 \\
\hline$n 3-5$ & F. CROCCHIO & Sersale & (Cl) & $19 / 7 / 85$ & - & 000 & 13.7 \\
\hline$n=-6$ & T. CAMPAMARO & Sersale & (Cl) & $18 / 7 / 95$ & - & 600 & 15.0 \\
\hline BJ-7 & F.IACINA & Roccaber narda & (Cl) & $17 / 7 / 85$ & - & 0 & - \\
\hline$n 3-8$ & T. DEL NOLIND & lagarise & (c) & $19 / 7 / 65$ & - & 450 & 13.6 \\
\hline A3 $3-9$ & T.SERAPITE & Verzino & (CL) & $22 / 7 / 8 b$ & ' & 450 & 17.5 \\
\hline $\mathrm{A3}-10$ & T. FALERKA & Falerna & (cl) & $27 / 7 / 36$ & " & 490 & 18.9 \\
\hline A3 -11 & T.S. BRUNELLO & Serra S. Bruno & ICl! & $28 / 7 / 8 b$ & - & 1100 & 12.9 \\
\hline n5 -12 & A4.STILARL & Bosto of Stilo & $(\mathrm{RC})$ & $29 / 7 / 86$ & • & 950 & 15.2 \\
\hline HJ -13 & T. STILARO & Bi vengi & (RC) & $30 / 7 / 96$ & - & 210 & 18.8 \\
\hline $14-14$ & T.M. SCORDA & Piano di tervo & (RC) & $31 / 7 / 36$ & - & 1350 & 14.5 \\
\hline$M J-15$ & F.R S.AGATA & Sella Entrata & (RC) & $1 / 8 / 86$ & - & 1350 & 14.7 \\
\hline $43-16$ & F.ra CRASTO & S, Efeciz Aspr. & $(\mathrm{RC})$ & $10 / 8 / 86$ & - & 450 & 17.7 \\
\hline H3 -17 & Af. AHENDOLEA & Roghudi & [RC] & 4/8/BO & - & 600 & 21.8 \\
\hline
\end{tabular}


11 = Longueur organe génitale.

12 - Largeur maximale organe génitale.

13 = Distance entre la partie terminale du génitale et le pore excréteur.

14 = Distance entre le génitale et la partie terminale du corps.

15 = Longueur infracapitulum.

$16=$ Longueur chélicères.

17 = Hauteur infracapitulum (à la base de l'articulation du palpe).

18 = Longueur dorsale $1^{\text {er }}$ article du palpe (P1).

$19=$ Longueur dorsale $2^{\star}$ article du palpe (P2).

$20=$ Longueur dorsale $3^{\mathrm{e}}$ article du palpe (P3).

$21=$ Longueur dorsale $4^{e}$ article du palpe (P4).

$22=$ Longueur dorsale $5^{\mathrm{e}}$ article du palpe (P5).

23 = Longueur maximale appareil éjaculateur (ejaculatory complex).

24 = Diamètre de la chambre proximale de l'appareil éjaculateur.

Certaines de ces valeurs ont été utilisées pour calculer les indices morphologiques suivants (en $\%$ ) :

$A=$ Largeur/longueur écusson dorsale (2/1).

$\mathrm{B}=$ Longueur plaque dorsale inférieur/antérieur $(6 / 5)$.

$\mathrm{C}=$ Longueur épinière II $+1 \mathrm{II} /$ épinière I (10/9).

$\mathrm{D}=$ Largeur/Longueur organe génitale (12/11).

$\mathrm{E}=$ Longueur P2/P4 (19/21).

$\mathrm{F}=$ Dist. génitale-extrémité du corps / Longueur ventrale $(14 / 3)$.

Les caractères examinés seront ensuite indiqués conformément aux numéros précédents.

\section{Les espèces recueillies}

\section{A. Sous-genre Torrenticola Piersig 1986}

A. 1. T. anomala (Koch 1837) (fig. 6 a-d)

$T$. longirostris (Szalay 1933 a), nov. syn.

T. piriformis Laska 1953, nov. syn.

Distribution géographique : holoarctique.

Ecologie : eurytherme, rhéophile.

Stations de récolte : $\mathrm{C} 2-1(2 \circ-\mathrm{m}-, 2$ - 2 - - , 1 nymphe - $\mathrm{n}-\mathrm{)}$; $\mathrm{C} 2-6$ (1 f) ; C2-8 (1 f) ; C2-13 $(3 \mathrm{~m}) ; \mathrm{C} 2-16(1 \mathrm{f}) ; \mathrm{M} 3-6(1 \mathrm{~m}, 1 \mathrm{f}) ; \mathrm{M} 3-12(1 \mathrm{f})$; M3-16 (1 m) ; M3-17 (2 m). Total : 16 individus.

En Italie, $T$. anomala a été signalé dans les Alpes aux sources de la Valle Anzasca (Monti 1910), dans le fleuve Ticino (Maglio 1905, 1907), dans l'Apennin central des fleuves Esino et Potenza (Maglio 1956), dans le fleuve Vomano (Cicolani \& Di Sabatino 1985 a) et dans le lac de Bracciano (Marucci 1906).
L'espèce se distingue bien des autres parce qu'elle présente chez l'exemplaire $\sigma$ un infracapitulum ventralement presque droit (fig. $6 \mathrm{c}$ ), une remarquable longueur des épimères II + III, un organe génital arrondi de petite dimension (fig. 6b) et une structure caractéristique de l'appareil éjaculateur (fig. 6d). Ces particularités sont aussi évidentes dans la description de T. longirostris (Szalay 1933a) et de $T$. piriformis (Laska 1953), morphologiquement semblables à $T$, anomala. Pour examiner la variabilité morphologique de l'espèce, une recherche biométrique a été effectuée sur 24 caractères sur tous les exemplaires disponibles ( $10 \sigma \square$ et $6 \%$ \%). Dans le tableau II, ont été reportées les valeurs moyennes, le rang de variabilité calculé et les données prises des descriptions originales de $T$. longirostris et de $T$. piriformis, les types n'ayant pu être examinés. La comparaison avec $T$. longirostris, relative à la $q$ met en évidence des différences dans quelques caractères dimensionnels $\left(\mathrm{n}^{\circ} 1-2-3-4\right)$ et une certaine uniformité avec ceux qui se réfèrent à l'unique exemplaire $q$ décrit, en Corse, par Angelier (1954) qui affirme: "Torrenticola longirostris appartient au groupe Torrenticola anomala... il s'agit cependant d'une espèce indépendante, par la forme du front (malgré les réserves que l'on doit faire sur la stabilité de celle-ci), droite et aux saillies des yeux et des mamelons chitineux portant des soies antenniformes peu accusées, et par les plaques dorsales latérales, très larges à leur extrémité postérieure ". Il apparaît clairement que la séparation des deux espèces, d'après les observations sur des exemplaires $Q$, a été faite sur des caractères peu stables comme on a pu le constater aussi dans notre étude qui nous a permis de remarquer la grande variabilité dans la forme de l'extrémité postérieure des plaques dorsales latérales. De telles différences perdent plus encore de signification lorsqu'on considere les indices morphologiques qui ne sont pas influencés par les tailles et l'âge des individus. Seul l'indice relatif aux plaques dorsales antérieures et postérieures (indice B, Tableau II) présente des différences dues à la variabilité accentuée du caractère.

La comparaison des valeurs des caractères des $\varnothing^{*}$ en notre possession avec celles relatives à la description du $\sigma$ faite par Laska (1953) révèle quelques différences dans la longueur des épimères II + III $\left(\mathrm{n}^{\circ}\right.$ 10 ), dans la distance entre la partie terminale du génitale et le pore excréteur $\left(n^{\circ} 13\right)$ et dans celle entre la zone génitale et la partie terminale de la superficie 
Tableau II. Valeurs moyennes (en $\mu \mathrm{m}$ ) des mesures des caractères et des indices morphologiques des mâles et femelles de $T$. anomala, $T$. longirostris et $T$. piriformis.

Entre parenthèses, le nombre d'observations et l'intervalle de variation.

\begin{tabular}{|c|c|c|c|c|c|c|}
\hline \multicolumn{3}{|c|}{ MALES } & \multicolumn{3}{|c|}{ FEMELLES } & \multirow[b]{2}{*}{$\begin{array}{r}\text { lofgirostris } \\
\text { 5zalay 19J3 }\end{array}$} \\
\hline car sp. & $\underset{1101}{\text { anowal }}$ & $\begin{array}{l}\text { longleastr15 } \\
\text { Laskz, } 1955\end{array}$ & $\begin{array}{c}\text { monal: } \\
(b)\end{array}$ & $\begin{array}{l}\text { pertifornis } \\
\text { Laskd 1953 }\end{array}$ & $\begin{array}{l}\text { longirostris } \\
\text { Angel.1854 }\end{array}$ & \\
\hline 11 & $\begin{array}{c}570 \\
(503-650)\end{array}$ & 552 & $\begin{array}{c}641 \\
(589-650)\end{array}$ & 655 & 620 & 750 \\
\hline 21 & $\begin{array}{c}462 \\
(399-522)\end{array}$ & 131 & $\begin{array}{c}502 \\
(15 b-535)\end{array}$ & 520 & 500 & 650 \\
\hline 31 & $\begin{array}{c}718 \\
(617-779)\end{array}$ & 669 & $\begin{array}{c}758 \\
(712-826)\end{array}$ & 840 & 160 & 934 \\
\hline 4 & $\begin{array}{c}534 \\
(494-617)\end{array}$ & 476 & $\begin{array}{c}585 \\
(532-630)\end{array}$ & 600 & 580 & 734 \\
\hline 5$)$ & $\begin{array}{c}186 \\
(1) 2-196)\end{array}$ & 179 & $\begin{array}{c}189 \\
(176-204)\end{array}$ & 160 & 186 & 217 \\
\hline b) & $\begin{array}{c}124 \\
(110-13)\end{array}$ & 131 & $\begin{array}{c}125 \\
\left(11^{7}-(1) !\right)\end{array}$ & $: 20$ & 112 & 167 \\
\hline$\pi$ & $\begin{array}{c}139 \\
(115-158)\end{array}$ & 131 & $\begin{array}{c}140 \\
(115-170)\end{array}$ & 190 & 118 & 150 \\
\hline B) & $\begin{array}{c}68 \\
|66-74|\end{array}$ & 73 & $\begin{array}{c}79 \\
(70-86)\end{array}$ & 75 & - & 100 \\
\hline 9) & $\begin{array}{c}293 \\
(251-335)\end{array}$ & $25 b$ & $\begin{array}{c}282 \\
\mid 254-320\}\end{array}$ & - & - & - \\
\hline 10) & $\begin{array}{c}142 \\
(153-15 b)\end{array}$ & 173 & $\begin{array}{c}6 b \\
(50-8 b)\end{array}$ & 60 & 70 & $b 7$ \\
\hline 111 & $\begin{array}{c}125 \\
(114-135)\end{array}$ & 131 & $\begin{array}{c}164 \\
(145-180)\end{array}$ & 180 & 140 & 175 \\
\hline 121 & $\begin{array}{c}107 \\
(90-11)]\end{array}$ & 110 & $\begin{array}{c}155 \\
(133-168)\end{array}$ & 160 & 130 & 175 \\
\hline 15) & $\begin{array}{c}111 \\
(95-133)\end{array}$ & 89 & $\begin{array}{c}179 \\
(137-196)\end{array}$ & 190 & - & 217 \\
\hline 16) & $\begin{array}{c}158 \\
{[117-19 b]}\end{array}$ & 109 & $\begin{array}{c}265 \\
\{212-305\}\end{array}$ & 320 & 312 & 350 \\
\hline 15) & $\begin{array}{c}308 \\
(262-361)\end{array}$ & 374 & $\begin{array}{c}32 b \\
{[305-356]}\end{array}$ & 315 & 286 & 353 \\
\hline (b) & $\begin{array}{c}336 \\
(290-372)\end{array}$ & 303 & $\begin{array}{c}360 \\
1337-4001\end{array}$ & 342 & 310 & 367 \\
\hline 171 & $\frac{86}{\mid 80-98)}$ & 90 & $\begin{array}{c}107 \\
1102-117 \pi\end{array}$ & 117 & 100 & 117 \\
\hline (19) & $\frac{33}{(30-55)}$ & 30 & $\begin{array}{c}35 \\
(34-36)\end{array}$ & 3 & 28 & 33 \\
\hline 191 & $\begin{array}{c}99 \\
(84-112\}\end{array}$ & 88 & $\begin{array}{c}105 \\
(97-12)\}\end{array}$ & 102 & 90 & 100 \\
\hline 20) & $\begin{array}{c}69 \\
(57-76)\end{array}$ & 60 & $\begin{array}{c}74 \\
(69-83)\end{array}$ & 68 & 68 & 75 \\
\hline 211 & $\begin{array}{c}106 \\
(92-1 \mid b)\end{array}$ & 95 & $\begin{array}{c}118 \\
(104-13)\end{array}$ & 113 & 105 & 117 \\
\hline 221 & $\begin{array}{c}19 \\
(18-21)\end{array}$ & 17 & $\frac{24}{(2 J-25)}$ & 25 & 17 & 20 \\
\hline 23) & $\begin{array}{c}208 \\
(196-22)]\end{array}$ & - & - & - & - & \\
\hline 241 & $\begin{array}{c}118 \\
(104-129)\end{array}$ & - & - & - & - & \\
\hline A & 79.9 & 78.0 & 78.3 & 79.3 & 91.2 & 60.6 \\
\hline 8 & 66.7 & 73.1 & 66.1 & 75.0 & 76.9 & 60.2 \\
\hline c & 48.4 & 67.5 & 23. & - & - & - \\
\hline 0 & 85.6 & 83.9 & 94.5 & 88.8 & 190.0 & 92.8 \\
\hline E & 95.1 & 92.8 & 86.9 & 90.2 & 85.1 & 85.7 \\
\hline F & 22.0 & 16.3 & 34.9 & 38.0 & 37.1 & 11.0 \\
\hline
\end{tabular}


ventrale $\left(n^{\circ} 14\right)$. Il est possible que l'individu examiné par Laska soit un exemplaire relativement jeune puisqu'il présente le pore excréteur proche de la partie terminale du corps et un accroissement réduit de la zone post-génitale ( $\mathrm{n}^{\circ} 14$ et indice $\mathrm{F}$ ), comme on l'observe sur les jeunes exemplaires étudiés par nous, chez lesquels ces caractères sont toujours associés à une plus grande longueur de la ligne de suture des épimères II et III $\left(n^{\circ} 10\right)$ par rapport à la longueur des épimères $I\left(n^{\circ} 9\right.$ et indice C). En outre, les caractères qualitatifs (forme du front et de la partie inférieure des plaques postérieures, forme de la superficie ventrale du P II et de la suture des IV épimères) invoqués par Szalay, présentent une grande variabilité, et on ne peut par conséquent reconnaître la validité spécifique de $T$. longirostris.

De même, $T$. piriformis, décrit sur une $\&$ (Laska 1953), doit être considéré comme synonyme de $T$. anomala. L'auteur, dans la description originale affirme : " Diese neue Form steht Torrenticola anomala Piersig am nächsten, unterscheidet sich aber durch den Palpenbau, die Form des Maxillarorgans und hauptsächlich durch die Körperform. " Tels caractères qualitatifs, actuellement considérés comme non discriminants par la plupart des spécialistes, la similitude des valeurs, relevée par nous (Tableau II) et l'absence du $\sigma$, encore inconnu, ne permettent pas de reconnaître la validité spécifique de $T$. piriformis.

A. 2. Torrenticola crenobia Di Sabatino \& Cicolani 1989 (fig. $6 \mathrm{e}-\mathrm{h}$ )

Distribution : Italie, Apennin méridional.

Ecologie : sténotherme, crénobionte.

Les exemplaires étudiés proviennent d'une station de l'Apennin méridional (M2-11). La capture de deux $\sigma \circ$ et de deux $\rho$ dans une localité située à quelques mètres de la source du torrent San Brunello $(\mathrm{CZ})$, semble confirmer que l'espece doit être considérée comme l'unique représentante typiquement crénobionte du genre (Di Sabatino \& Cicolani 1989). $T$. crenobia, comme on le deduit des données du tableau III relatives à un $\sigma^{*}$ et une $q$, est bien caractérisée morphologiquement dans les deux sexes, se distinguant de $T$. anomala par certains caractères dimensionnels et par tous les indices morphologiques considérés (Tableau VIII). L'analyse des caractères qualitatifs et les différentes exigences écologiques discutées dans la description de l'espèce confirment la validité spécifique de $T$, crenobia.

\section{A. 3. Torrenticola elliptica (Maglio 1909) (fig. $6 \mathrm{i}-\mathrm{n}$ )}

Distribution : euroasiatique.

Ecologie : sténotherme et rhéobionte.

Stations: $\mathrm{Cl}-2(1 \mathrm{~m}, 3 \mathrm{f})$; $\mathrm{C} 2-6(1 \mathrm{~m})$; $\mathrm{C} 2.7$ (1 f) ; C2-11 (16 m, 34 f, $5 \mathrm{n})$; C2-12 (55 m, $45 \mathrm{f}$, $2 \mathrm{n})$; C2-13 (36 m, $18 \mathrm{f}, 2 \mathrm{n})$; C2-14 (1 m) ; M2-3 ( $1 \mathrm{~m}, 2 \mathrm{f}$ ). Total : 223 individus.

En Italie, l'espèce était connue dans le Trentin Haute-Adige (Maglio 1909) et dans le milieu hyporhéique du torrent S. Bernardino-lac de Garde (Schwoerbel 1961).

L'étude biométrique (Tableau IV), effectuée sur $10 \circ \sigma^{\circ}$ et 10 우 a permis de distinguer, chez les $\sigma$, a) une faible valeur du rapport largeur/longueur de l'écusson dorsal (indice A), qui confirme ce qui a été signalé par d'autres auteurs, considérant $T$. elliptica comme une espèce typiquement " mince » (fig. 6, i) ; b) un organe génital déplacé antérieurement (indice $F$ et fig. 6,1 ) ; c) une valeur assez constante dans le rapport longueur épimères II + III /

Tableau III. Mesures des caractères (en $\mu \mathrm{m}$ ) d'un mäle $(\mathbf{M})$ et d'une femelle (F) de $T$. crenobia.

\begin{tabular}{|c|c|c|c|c|c|c|c|c|}
\hline Ear. & $M$ & $\mathbf{F}$ & car. & M & $F$ & car. & $M$ & $F$ \\
\hline 1) & 693 & 750 & 93 & 325 & 325 & ${ }^{-171}$ & 110 & 145 \\
\hline 2) & 503 & 508 & 101 & 173 & 35 & 18) & 33 & 40 \\
\hline 3) & B17 & 855 & (11) & 156 & 176 & 19) & 112 & 112 \\
\hline 4) & 608 & 636 & 121 & 121 & 180 & 20) & 64 & 65 \\
\hline 5) & 235 & 217 & 1.3) & 129 & 219 & 211 & 108 & 109 \\
\hline 6) & 1.57 & 152. & 14) & 172 & 312 & 22) & 20 & 20 \\
\hline 7) & 170 & $17 \bar{t}$ & 15) & 360 & 333 & 23) & 250 & - \\
\hline 8) & 90 & 102 & 16) & 420 & 390 & 24) & 109 & - \\
\hline
\end{tabular}


Tableau IV. Valeurs moyennes en $\mu \mathrm{m}$ ) des mesures des caractères et des indices morphologiques des mâles et des femelles de $T$. elliptica, $T$. similis, $T$. meridionalis et $T$. microphallus. Entre parenthèses, le nombre d'observations et l'intervalle de variation.

MALES FEFELLES

\begin{tabular}{|c|c|c|c|c|c|c|c|c|}
\hline $\operatorname{car}^{\text {esp. }}$ & $\begin{array}{l}\text { Elliptica } \\
(10)\end{array}$ & $\begin{array}{l}\text { sicillis } \\
\text { prep. 290 }\end{array}$ & $\begin{array}{l}\text { seridional is } \\
\{10\}\end{array}$ & $\begin{array}{l}\text { i crophal lus } \\
\text { Lundblad, } 1956\end{array}$ & $\underset{(10)}{\text { elliptica }}$ & $\begin{array}{l}\text { sinilis } \\
\text { prep.29l }\end{array}$ & $\begin{array}{l}\text { Aeridionalis } \\
\qquad(10)\end{array}$ & $\begin{array}{l}\text { eicrophallus } \\
\text { Lundtal ad, } 5956\end{array}$ \\
\hline 11 & $\begin{array}{c}758 \\
(703-807)\end{array}$ & 798 & $\begin{array}{c}660 \\
(608-693)\end{array}$ & 636 & $\begin{array}{c}769 \\
(731-826)\end{array}$ & 850 & $\begin{array}{c}714 \\
(686-74])\end{array}$ & 761 \\
\hline 2) & $\begin{array}{c}470 \\
\{456-513\}\end{array}$ & 503 & $\begin{array}{c}159 \\
(437-465)\end{array}$ & 427 & $\begin{array}{c}195 \\
(456-532)\end{array}$ & 550 & $\begin{array}{c}532 \\
{[48+-636)}\end{array}$ & 532 \\
\hline 3) & $\begin{array}{c}839 \\
(779-874)\end{array}$ & 931 & $\begin{array}{c}784 \\
(760-817)\end{array}$ & 760 & $\begin{array}{c}887 \\
(817-950)\end{array}$ & 975 & $\begin{array}{c}951 \\
(779-902)\end{array}$ & 874 \\
\hline 4) & $\begin{array}{c}592 \\
(560-646)\end{array}$ & 598 & $\begin{array}{c}532 \\
(513-560)\end{array}$ & 490 & $\begin{array}{c}618 \\
(579-674)\end{array}$ & 675 & $\begin{array}{c}620 \\
(570-616)\end{array}$ & 617 \\
\hline 5) & $\begin{array}{c}213 \\
(196-235)\end{array}$ & 203 & $\begin{array}{c}203 \\
(186-211)\end{array}$ & 210 & $\begin{array}{c}217 \\
(203-235)\end{array}$ & 259 & $\begin{array}{c}215 \\
(199-22])\end{array}$ & 215 \\
\hline 6) & $\begin{array}{c}132 \\
(12 !-141)\end{array}$ & 137 & $\begin{array}{c}131 \\
(125-137)\end{array}$ & 175 & $\begin{array}{c}143 \\
(133-(52)\end{array}$ & 168 & $\begin{array}{c}142 \\
(13)-(15)\end{array}$ & 145 \\
\hline 7) & $\begin{array}{c}147 \\
(140-156)\end{array}$ & 169 & $\begin{array}{c}164 \\
(154-172)\end{array}$ & 156 & $\begin{array}{c}176 \\
(16 b-185)\end{array}$ & 196 & $\begin{array}{c}187 \\
(17)-193)\end{array}$ & 203 \\
\hline B) & ${ }^{94}$ & 94 & $\begin{array}{c}85 \\
(78-94)\end{array}$ & 76 & $\begin{array}{c}99 \\
(96-105)\end{array}$ & 117 & $\begin{array}{c}97 \\
(93-102)\end{array}$ & 98 \\
\hline 9) & $\begin{array}{c}306 \\
(290-309)\end{array}$ & 333 & $\begin{array}{c}309 \\
(298-325)\end{array}$ & 318 & $\begin{array}{c}342 \\
(325-350)\end{array}$ & 395 & $\begin{array}{c}334 \\
(321-348)\end{array}$ & $32 !$ \\
\hline 10) & $\begin{array}{c}109 \\
(102-111)\end{array}$ & 117 & $\begin{array}{c}95 \\
(87-98)\end{array}$ & 135 & $\begin{array}{c}47 \\
(35-51)\end{array}$ & $5 !$ & $\begin{array}{c}34 \\
(27-47)\end{array}$ & 40 \\
\hline 11) & $\begin{array}{c}177 \\
{[172-189]}\end{array}$ & 190 & $\begin{array}{c}162 \\
(15 b-172)\end{array}$ & 157 & $\begin{array}{c}193 \\
(176-20)\end{array}$ & $20 \mathrm{~J}$ & $\begin{array}{c}179 \\
(172-180)\end{array}$ & 190 \\
\hline 121 & $\begin{array}{c}136 \\
(133-1+5)\end{array}$ & 140 & $\begin{array}{c}13 ! \\
{[125-137\}}\end{array}$ & 114 & $\begin{array}{c}168 \\
(152-186)\end{array}$ & 198 & $\begin{array}{c}167 \\
(15 b-17 b)\end{array}$ & 165 \\
\hline 13) & $\begin{array}{c}168 \\
(156-196)\end{array}$ & 178 & $\begin{array}{c}172 \\
(156-189)\end{array}$ & 117 & $\begin{array}{c}223 \\
(196-254)\end{array}$ & 294 & $\begin{array}{c}217 \\
(20)-235)\end{array}$ & 215 \\
\hline 14) & $\begin{array}{c}224 \\
(204-243)\end{array}$ & 276 & $\begin{array}{c}215 \\
(200-230)\end{array}$ & $15 t$ & $\begin{array}{c}306 \\
(250-360)\end{array}$ & 100 & $\begin{array}{c}290 \\
(227-316)\end{array}$ & 315 \\
\hline 15) & $\begin{array}{c}312 \\
(290-33))\end{array}$ & 305 & $\begin{array}{c}306 \\
{[202-316]}\end{array}$ & 282 & $\begin{array}{c}336 \\
1330-350]\end{array}$ & $3 B 0$ & $\begin{array}{c}330 \\
(323-311)\end{array}$ & 332 \\
\hline 16) & $\begin{array}{c}352 \\
{[329-356]}\end{array}$ & 337 & $\begin{array}{c}347 \\
(331-360)\end{array}$ & 330 & $\begin{array}{c}380 \\
(372-392\}\end{array}$ & $\$ 10$ & $\begin{array}{c}387 \\
(380-399)\end{array}$ & 388 \\
\hline 17) & $\begin{array}{c}125 \\
(117-137)\end{array}$ & 137 & $\begin{array}{c}122 \\
(117-129)\end{array}$ & 117 & $\begin{array}{c}141 \\
(137-(45)\end{array}$ & - & $\begin{array}{c}13 b \\
(128-[45)\end{array}$ & - \\
\hline 18) & $\begin{array}{c}56 \\
(35-60)\end{array}$ & 31 & $\begin{array}{c}34 \\
\mid 30-35)\end{array}$ & 34 & $\begin{array}{c}39 \\
(38-40)\end{array}$ & 40 & $\begin{array}{c}30 \\
(39-40)\end{array}$ & 36 \\
\hline 191 & $\begin{array}{c}100 \\
(95-112)\end{array}$ & 100 & $\begin{array}{c}97 \\
(95-100]\end{array}$ & 95 & $\begin{array}{c}109 \\
(104-(16)\end{array}$ & 123 & $\begin{array}{c}110 \\
(104-114)\end{array}$ & 109 \\
\hline 201 & $\begin{array}{c}59 \\
\{50-62\}\end{array}$ & 57 & $\begin{array}{c}58 \\
(56-60)\end{array}$ & 52 & (b2-6b\} & 71 & $\begin{array}{c}64 \\
(60-66)\end{array}$ & 64 \\
\hline 21) & $\begin{array}{c}100 \\
(75-114)\end{array}$ & 97 & $\begin{array}{c}97 \\
(95-100)\end{array}$ & จง & $\begin{array}{c}107 \\
|97-115\rangle\end{array}$ & 116 & $\begin{array}{c}108 \\
(104-109]\end{array}$ & 105 \\
\hline 221 & $\begin{array}{c}20 \\
(21-23)\end{array}$ & 21 & $\begin{array}{c}20 \\
\mid 20-22)\end{array}$ & 20 & $\begin{array}{c}22 \\
(20-22)\end{array}$ & 22 & $\begin{array}{c}22 \\
(21-22)\end{array}$ & 22 \\
\hline 23) & $\begin{array}{c}200 \\
(270-206)\end{array}$ & 202 & $\begin{array}{c}250 \\
(244-256)\end{array}$ & 101 & & & & \\
\hline 2414 & 125 & 130 & 137 & 78 & & & & \\
\hline A & 63.7 & 63.8 & 69.5 & 67.1 & 64.4 & 64.7 & 74.5 & 69.9 \\
\hline 8 & 61.9 & 67.5 & 64.8 & 59.5 & 65.9 & 64.8 & 66.1 & 67.4 \\
\hline c & 35.7 & 35.1 & 31.0 & 11.8 & 13.7 & 12,9 & 10.2 & 12,4 \\
\hline 0 & 76.7 & 73.7 & 00.7 & 72.6 & 07.0 & 92.6 & 93.3 & 88.8 \\
\hline E & 100.0 & 103.0 & 100.0 & 100.0 & 102.0 & 106.0 & 102.0 & 103.8 \\
\hline$F$ & 26.8 & 29.6 & 27.4 & 20.5 & 34.5 & 30.1 & 34.0 & 36.0 \\
\hline
\end{tabular}


longueur épimères I (indice $C$ ) ; d) une chambre proximale de l'appareil éjaculateur bien développée (fig. $6 \mathrm{n}$ ).

Chez les $९$, les valeurs relatives de la forme de l'écusson dorsal (indice $\mathrm{A}=64,4 \%$ ) et de la forme du génital ( $D=87 \%$ ) présentent simplement une légère variation des valeurs moyennes, démontrant une certaine stabilité des caractères.

Il semble qu'une espèce affine à $T$. elliptica soit T. microphallus Lundblad 1956 considérée sousespèce par Viets (1967). L'examen du matériel typique de T. microphallus (prép. $n^{\circ} 4049$ or et 4027 $\varnothing$ du Musée de Stockholm) et celui que nous avons déterminé comme $T$. elliptica (Tableau IV) nous a permis de mettre en évidence dans le sexe $\sigma$ des différences dimensionnelles dans les rapports largeur/longueur de l'écusson dorsal, largeur/longueur de l'organe génital, longeur des épimères II + III / épimères I, dans les dimensions de l'appareil éjaculateur et dans l'extension de la zone post-génitale $(\mathrm{F}=20,5$ vs $26,8 \%)$.

La distinction du sexe $\supsetneq$ est plus difficile, comme c'est fréquent chez toutes les espèces du genre.

L'examen biométrique et les observations du matériel typique incitent à retenir valable

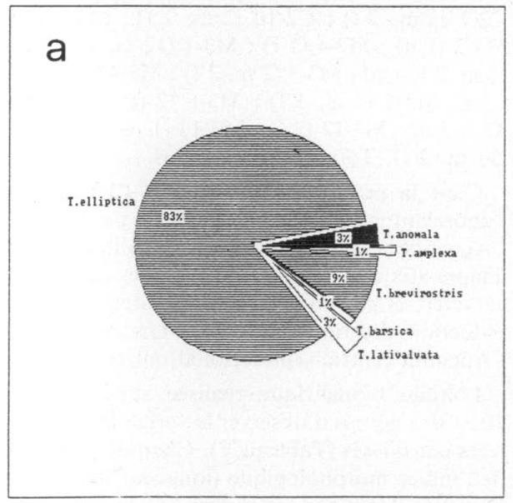

T. microphallus. Il résulte des données rapportées par Lundblad (1956) qu'elle est caractérisée même écologiquement en Espagne en présentant une distribution altimétrique plus large $(500-2300 \mathrm{~m})$, contrairement à $T$. elliptica qui semble confinée dans des stations au-dessus de $900 \mathrm{~m}$. Un examen attentif du matériel recueilli par Lundblad montre aussi une sympatrie dans une station de la Sierra Nevada située à $2300 \mathrm{~m}$ et, par conséquent, l'absence du flux génétique entre les deux entités reconnues morphologiquement par l'auteur.

T. elliptica, qui semble être bien distribuée en Italie, est particulièrement abondante dans l'Apennin des Abruzzes entre 300 et $1100 \mathrm{~m}$ où elle représente $83 \%$ des individus recueillis (fig. 3). La sténothermie de l'espèce est évidente d'après les données reportées sur la figure 5 .

\section{A. 4. Torrenticola similis (Viets 1939) (fig. 6 o-r)}

Distribution : Pyrénées, Alpes, Pologne.

Ecologie : sténotherme, rhéobionte, occasionne]lement interstitiel.

Notre découverte dans une station de l'Apennin septentrional S2-1 $(1 \mathrm{~m}, 1 \mathrm{f})$, est la première pour l'Italie. La position typique du pore excréteur sur la zone de sclérotisation primaire (fig. $6 \mathrm{p})$ a été individualisée sur les deux exemplaires examinés, même

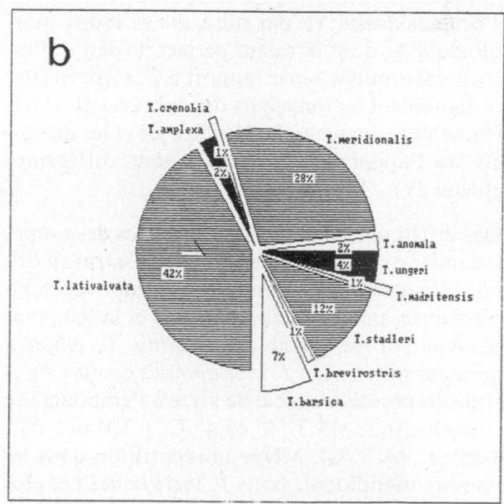

Fig. 3. Abondance relative des espèces dans l'Apennin abruzzain (a) et calabrais (b). 
si une telle particularité apparaît chez un jeune individu de sexe $\sigma$ appartenant à une population de $T$. elliptica. Les autres caractères longueur du capitulum $\left(n^{\circ} 7\right)$, longueur des épimères I $\left(n^{\circ} 9\right)$ et la plus grande ext ension de la zone post-génitale (indice F) semblent avoir une importance au niveau spécifique par rapport à $T$. elliptica. De plus, l'examen de l'appareil éjaculateur montre des différences évidentes, avec une chambre proximale très réduite et un relief antérieur plus développé (fig. $6 \mathrm{r}$ ).

\section{A. 5. Torenticola meridionalis Di Sabatino \& Cico- lani 1990 (fig. 7 a-d)}

Distribution : Apennin Calabrais.

Ecologie : eurytherme.

Stations: M3-2 (1 f) ; M3-3 (2 m, $4 \mathrm{f})$ : M3-5 $(2 \mathrm{~m}, 1 \mathrm{n})$; M3-6 (6m, $2 \mathrm{f}) ; \mathrm{M} 3-8(2 \mathrm{~m}, 4 \mathrm{f})$; M3-10 (6 m, 10 f, 3 n) ; M3-11 (4 m, 6 f); M3-12 (1 m, $4 \mathrm{f}) ; \mathrm{M} 3-13$ (2 f) ; M3-14 (1 f) ; M3-15 (9 m, $10 \mathrm{f})$; M3-16 (6 m, $3 \mathrm{f})$. Total : 94 individus.

Les stations sont toutes localisées dans l'Apennin calabrais où sont relativement nombreux les représentants de cette espèce décrite par Sabatino \& Cicolani (1990) sur du matériel provenant du fleuve Alli (CZ). Comme on peut le déduire du Tableau IV, les affinités avec $T$. elliptica sont considérables puisque la comparaison des données dimensionnelles et morphologiques, effectuées sur 10 ơ ot $10 \%$ \%, révèlent des différences seulement dans les dimensions de l'écusson dorsal, et, par suite, dans l'indice morphologique A, dont la valeur permet de définir l'espèce moins " mince" par rapport à $T$. elliptica (fig. 7 a). Egalement les longueurs des épimères II + III, la forme de l'organe génital (indice D) et les dimensions de l'appareil éjaculateur, sont différentes (Tableau IV).

Des différences significatives entre les deux espèces, comme on l'a déjà remarqué dans le travail original, concernent quelques caractéristiques écologiques comme, par exemple, l'altitude et la température. Ainsi, il est possible de définir $T$. elliptica comme sténotherme et $T$. meridionalis comme eurytherme et euryèce, capable de vivre à l'embouchure des fleuves (stat. M3-3: $\mathrm{t}^{\circ} 29,4^{\circ} \mathrm{C}, 1350 \mathrm{~m}$; stat. M3-15: $t^{\circ} \quad 14,7^{\circ} \mathrm{C}$ ). Même la répartition dans les Apennins (méridionale pour $T$. meridionalis et plus large pour $T$. elliptica) pourrait résulter de différentes exigences écologiques. Dans l'A Apennin calabrais, le régime des cours d'eau (crues imprévues alternant avec une extrême réduction de débit) provoque, dans la partie inférieure, des lits détritiques très larges avec une diversification de niches due à une typologie fluviale très fréquente dans certaines zones de la région méditerranéenne (Gerecke 1986).

L'entité géomorphologique de l'Apennin calabrais pourrait ainsi expliquer l'abondance relative de l'espèce $(28 \%, f i g .3)$ et son association avec $T$. lativalvata et $T$. stadleri (Tableau IX) qui paraissent posséder de semblables exigences écologiques.

La problématique taxonomique existante entre ces espèces semble analogue à celle déjà indiquée pour $T$. elliptica et $T$. microphatlus. Elle pourrait être déterminée par les conditions des microhabitats dans la partie méridionale de l'aire de $T$. elliptica.

L'importance des facteurs écologiques qui nous ont conduit à séparer les deux espèces pourra être comparée à la lumière des résultats des recherches électrophorétiques sur les systèmes gène-enzyme (en cours de réalisation) déjà expérimentées en acarologie pour discriminer des espèces jumelles (Cicolani et al. 1981).

\section{A. 6. Torrenticola lativalvata Viets 1952 (Fig. $7 \mathrm{e}-\mathrm{h}$ )}

Distribution : Algérie, Maroc, Corse, Tchécoslovaquie, Bulgarie.

Ecologie : rhéobionte.

Stations : S2-2 (1 m, $1 \mathrm{f}) ; \mathrm{Cl}-1$ (1 f ; C2-4 (1 f) : C2-7 (2 m, $2 \mathrm{f})$; C2-10 (2 m, $2 \mathrm{f})$; M2-1 (2 f) ; M2-2 (1 n) ; M2-4 (1 f) ; M3-1 (12 m, $7 \mathrm{f})$; M3-2 $(4 \mathrm{~m}, 2 \mathrm{f}, 1 \mathrm{n}) ; \mathrm{M} 3-3(2 \mathrm{~m}, 2 \mathrm{f}) ; \mathrm{M} 3-4(32 \mathrm{~m}, 27 \mathrm{f}$, $2 \mathrm{n})$; M3-5 (3 m, $5 \mathrm{f})$; M3-6 (2f); M3-8 (2 m, $17 \mathrm{f}, \mathrm{l} \mathrm{n}) ; \mathrm{M} 3-12(2 \mathrm{f}) ; \mathrm{M} 3-13(\mathrm{~lm}, 2 \mathrm{f}): \mathrm{M} 3-17$ (11 $\mathrm{m}, 2 \mathrm{f})$. Total : 156 individus.

C'est la première citation pour l'Italie malgré l'abondant matériel (42\%) recueilli par nous dans l'Apennin calabrien où il semble qu'il se soit bien adapté aux conditions hydrologiques des biotopes caractérisés par un substrat caillouteux et par une réduction drastique du débit. Les récoltes dans l'Apennin central-septentrional ont été rares.

L'étude biométrique réalisée sur $10 \% \sigma$ et 10 ९ $\bigcirc$ a permis d'observer la variabilité des caractères considérés (Tableau V). Chez les $\sigma$ la valeur de l'indice morphologique (longueur des épimères II + III / longueur des épimères I) caractérise bien l'espèce ( $\mathrm{C}=46,4 \%)$. Etant donné l'abondance avec laquelle l'espéce a été recueillie en Italie 
Tableau V. Valeurs moyennes (en $\mu \mathrm{m}$ ) des mesures des caractères et des indices morphologiques de $T$. lativalvata

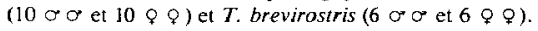

MALES

FEMELLES

\begin{tabular}{|c|c|c|c|c|}
\hline earact. & $\begin{array}{c}\text { lativaluate } \\
\text { (10) }\end{array}$ & $\begin{array}{l}\text { (b) } \\
\text { (b) }\end{array}$ & $\begin{array}{c}\text { lativaluta } \\
\text { (10) }\end{array}$ & $\begin{array}{l}\text { brevi rostris } \\
\text { (b) }\end{array}$ \\
\hline 1 & $\begin{array}{c}637 \\
1598-674)\end{array}$ & $\begin{array}{c}699 \\
(665-760)\end{array}$ & $(686-836)$ & $\begin{array}{c}788 \\
(760-836)\end{array}$ \\
\hline 2 & $\begin{array}{c}443 \\
(418-675)\end{array}$ & $\begin{array}{c}538 \\
(510-589\end{array}$ & $\begin{array}{c}520 \\
(494-598)\end{array}$ & $\begin{array}{c}623 \\
(600-665)\end{array}$ \\
\hline 3 & $\begin{array}{c}764 \\
(7\{2-826)\end{array}$ & $\begin{array}{c}821 \\
(779-864)\end{array}$ & $\begin{array}{c}894 \\
\{836-970)\end{array}$ & $\begin{array}{c}910 \\
(874-750)\end{array}$ \\
\hline 1 & $\begin{array}{c}512 \\
(5 ! 3-589)\end{array}$ & 684 & $\begin{array}{c}681 \\
(600-751)\end{array}$ & $\begin{array}{c}776 \\
(760-807)\end{array}$ \\
\hline 5 & $\begin{array}{c}206 \\
(200-213)\end{array}$ & $\begin{array}{c}199 \\
(192-211)\end{array}$ & $\begin{array}{c}237 \\
(215-24 T)\end{array}$ & $\begin{array}{c}200 \\
(196-203)\end{array}$ \\
\hline 6 & $\begin{array}{c}12 b \\
\mid\{2|-| 37 \mid\end{array}$ & $\begin{array}{c}151 \\
(14 !-160)\end{array}$ & $\begin{array}{c}150 \\
(145-(56)\end{array}$ & $\begin{array}{c}155 \\
(153-160)\end{array}$ \\
\hline 1 & $\begin{array}{c}153 \\
\{12 b-166\}\end{array}$ & $\begin{array}{c}136 \\
(133-137)\end{array}$ & $\begin{array}{c}190 \\
(170-205)\end{array}$ & $\begin{array}{c}16,2 \\
(145-17 b)\end{array}$ \\
\hline 8 & $\begin{array}{c}80 \\
(70-90\}\end{array}$ & $\begin{array}{c}94 \\
(90-99)\end{array}$ & $\begin{array}{c}109 \\
(105-117)\end{array}$ & $\begin{array}{c}96 \\
(78-94)\end{array}$ \\
\hline 9 & $\begin{array}{c}310 \\
(294-329)\end{array}$ & $\begin{array}{c}303 \\
(286-317)\end{array}$ & $\begin{array}{c}348 \\
(325-389)\end{array}$ & $\begin{array}{c}336 \\
(317-353)\end{array}$ \\
\hline 10 & $\frac{144}{(13]-[19)}$ & $\begin{array}{c}115 \\
(109-117)\end{array}$ & $\left(\begin{array}{c}41 \\
(3-51)\end{array}\right.$ & $\begin{array}{c}21 \\
(10-43)\end{array}$ \\
\hline 11 & $\begin{array}{c}150 \\
(14 t-15 b)\end{array}$ & $\begin{array}{c}191 \\
(104-200)\end{array}$ & $\begin{array}{c}190 \\
(184-199)\end{array}$ & $\begin{array}{c}198 \\
(180-211)\end{array}$ \\
\hline 12 & $\begin{array}{c}125 \\
(117-133)\end{array}$ & $\begin{array}{c}157 \\
(149-164)\end{array}$ & $\begin{array}{c}180 \\
(176-189)\end{array}$ & $\begin{array}{c}187 \\
(176-196)\end{array}$ \\
\hline 13 & $\begin{array}{c}110 \\
(98-145)\end{array}$ & 137 & $\begin{array}{c}241 \\
(196-274)\end{array}$ & $\begin{array}{c}245 \\
\{260-254\}\end{array}$ \\
\hline 14 & $\begin{array}{c}148 \\
(1,7-176)\end{array}$ & 235 & $\begin{array}{c}310 \\
(2+5-360)\end{array}$ & $\begin{array}{c}340 \\
(325-356)\end{array}$ \\
\hline 15 & $\begin{array}{c}302 \\
(294-317)\end{array}$ & $\begin{array}{c}284 \\
(274-294)\end{array}$ & $\begin{array}{c}358 \\
(345-362)\end{array}$ & $\begin{array}{c}318 \\
(305-333)\end{array}$ \\
\hline 16 & $\begin{array}{c}347 \\
(317-392)\end{array}$ & $\begin{array}{c}330 \\
(312-348)\end{array}$ & $\begin{array}{c}102 \\
(392-110)\end{array}$ & $\begin{array}{c}372 \\
(360-384)\end{array}$ \\
\hline 17 & $\stackrel{112}{(95-[3])}$ & $\begin{array}{c}117 \\
\text { (1) } 0-125)\end{array}$ & $\begin{array}{c}153 \\
(150-168)\end{array}$ & $\begin{array}{c}13 \delta \\
(130-160)\end{array}$ \\
\hline 18 & $\begin{array}{c}3 i \\
28-35\end{array} \mid$ & $\begin{array}{c}34 \\
\{3 J-35\}\end{array}$ & $\left(\begin{array}{c}40 \\
(59-40)\end{array}\right)$ & 35 \\
\hline 19 & $\begin{array}{c}100 \\
(93-112)\end{array}$ & $\begin{array}{c}92 \\
(85-100)\end{array}$ & $\begin{array}{c}120 \\
(109-130)\end{array}$ & $\begin{array}{c}101 \\
(90-107)\end{array}$ \\
\hline 20 & $\begin{array}{c}58 \\
(5 b-60)\end{array}$ & $\begin{array}{c}64 \\
(59-69)\end{array}$ & $\begin{array}{c}70 \\
(69-72)\end{array}$ & $\begin{array}{c}68 \\
(60-71)\end{array}$ \\
\hline 21 & $\begin{array}{c}99 \\
(95-108)\end{array}$ & $\begin{array}{c}104 \\
(97-109)\end{array}$ & $\begin{array}{c}115 \\
(104-119)\end{array}$ & $\stackrel{115}{(100-119)}$ \\
\hline 22 & $\begin{array}{c}20 \\
(20-22)\end{array}$ & $\begin{array}{c}30 \\
(2 B-31)\end{array}$ & $\left|\begin{array}{c}22 \\
\mid 20-22\end{array}\right|$ & $\begin{array}{c}32 \\
(30-33)\end{array}$ \\
\hline $23 *$ & 223 & 315 & - & - \\
\hline $24+$ & 86 & 105 & - & - \\
\hline A & 69.5 & 77.0 & 67.2 & 79.0 \\
\hline 8 & 61.1 & 75.5 & 6J.J & 77.5 \\
\hline c & 16.4 & 37.8 & 11.8 & 6.2 \\
\hline$j$ & 83.3 & 12.0 & 94.7 & 94.4 \\
\hline E & 101.0 & 89.4 & 104.3 & 69.3 \\
\hline$F$ & 19.5 & 30.1 & 34.6 & 37.4 \\
\hline
\end{tabular}

méridionale et sa présence en Afrique du Nord et en Corse, il est possible de considérer cette espèce typique du bassin méditerranéen, même si des études ultérieures peuvent préciser les limites septentrionales de son aire de répartition.

Le tableau IX montre dans l'Apennin central les cas les plus fréquents d'association avec $T$. brevirostris, tandis que dans l'A pennin méridional l'espèce est fréquemment associée avec $T$. stadleri et $T$. meridionalis.

\section{A. 7. Torrenticola barsica (Szalay 1933) (fig. $7 \mathrm{i}-\mathrm{n}$ ) Torrenticola mucranata Viets 1955, nov. syn.}

Stations : C2-9 (1 f) ; C2-10 (2 m, $1 \mathrm{f})$; M1-1 (1 f) ; M1-2 (1 f) ; M3-1 (3 m, 1 f) ; M3-3 (2 m, $2 \mathrm{f})$; M3-4 (3 m, $2 \mathrm{f})$; M3-5 (2 m, $2 \mathrm{f})$; M3-7 (1 m, $1 \mathrm{f})$; M3-8 (1 f) ; M3-9(2 f). Total : 28 individus.

Distribution : Corse, Espagne, France, Autriche, Tchécoslovaquie, Pologne, Hongrie, Bulgarie, Yougoslavie.

Ecologie : eurytherme, pétricole.

La découverte de l'espèce dans les stations de l'Apennin central et méridional, à des altitudes supérieures à $800 \mathrm{~m}$, est la première pour la faune italienne. $T$, barsica particulièrement abondante dans l'Apennin calabrais n'a jamais été recueillie dans les stations à température inférieure à $13^{\circ} \mathrm{C}$.

L'analyse biométrique (Tableau VI) a mis en évidence chez les $o$ quelques particularités qui peuvent avoir leur importance, comme la valeur du rapport largeur/longueur de l'organe génital (indice $\mathrm{D}=$ $88,7 \%$ ), la valeur presque constante des indices des épimères II + III / épimères I ( fig. 4) et, dans les deux sexes les dimensions du second article des palpes ( $\mathrm{P} 2)$ toujours plus grand du $\mathbf{P 4}$ (indice $\mathrm{E}=$ $110 \%$ ). Comme caractères qualificatifs on a observé un infracapitulum de forme et de dimension variable et, dans le sexe $\sigma$, deux glandes anales pas toujours au-dessous du pore excréteur mais aussi sur la même ligne.

L'appareil éjaculateur est bien caractérisé par deux bras proximaux déplacés vers le haut, par des bras distaux assez robustes, par une cavité proximale réduite et par un relief antérieur bien accentué (fig. $7 \mathrm{n}$ ).

La recherche a donné l'opportunité d'effectuer des comparaisons avec les espèces affines $T$. hispanica et $T$. mucronata. Lundblad (1956) dans sa 
Tableau VI. Valeurs moyennes ( $\mathrm{en} \mu \mathrm{m}$ ) des mesures des caractères et des indices morphologiques de $T$. barsica, T. hispanica et $T$. mucronata. Entre parenthèses, le nombre des observations et l'intervalle de variation.

\begin{tabular}{|c|c|c|c|c|c|c|}
\hline \multicolumn{4}{|c|}{ MALES } & \multicolumn{2}{|c|}{ FEMELLES } & \multirow[b]{2}{*}{$\begin{array}{l}\text { sucronata } \\
\text { K. Viets } 5\end{array}$} \\
\hline caract. & $\begin{array}{l}\text { barsica } \\
\text { (b) }\end{array}$ & $\begin{array}{l}\text { hispanica } \\
\text { Lundblad } 5 b\end{array}$ & $\begin{array}{l}\text { mucronak } \\
\text { K. Viets } 55\end{array}$ & $\begin{array}{l}\text { barsica } \\
\text { (6) }\end{array}$ & $\begin{array}{c}\text { hispanica } \\
\text { Lundblad } 1956\end{array}$ & \\
\hline 11 & $\begin{array}{c}614 \\
(570-636)\end{array}$ & 541 & 627 & $\begin{array}{c}691 \\
(674-722)\end{array}$ & 655 & 760 \\
\hline 2) & $\begin{array}{c}456 \\
(437-475)\end{array}$ & 418 & 465 & $\begin{array}{c}512 \\
(484-540)\end{array}$ & 550 & 570 \\
\hline 31 & $\begin{array}{c}742 \\
(693-779)\end{array}$ & 674 & 750 & $\begin{array}{c}858 \\
(826-893)\end{array}$ & 826 & 874 \\
\hline 4) & $\begin{array}{c}586 \\
(503-693)\end{array}$ & 475 & 522 & $\begin{array}{c}598 \\
(580-600)\end{array}$ & 617 & 627 \\
\hline 5) & $\begin{array}{c}179 \\
\mid 172-186\}\end{array}$ & 181 & 198 & $\begin{array}{c}201 \\
(196-211)\end{array}$ & 215 & 211 \\
\hline b) & $\begin{array}{c}137 \\
(133-141)\end{array}$ & 117 & 137 & $\begin{array}{c}139 \\
(137-149)\end{array}$ & 145 & 140 \\
\hline$n$ & $\begin{array}{c}194 \\
(172-200)\end{array}$ & 164 & 168 & $\begin{array}{c}214 \\
\{203-223\}\end{array}$ & 188 & 184 \\
\hline 8) & $\begin{array}{c}67 \\
(78-95)\end{array}$ & 74 & 94 & $\begin{array}{c}103 \\
(90-117)\end{array}$ & 90 & 105 \\
\hline 9) & $\begin{array}{c}307 \\
(282-321)\end{array}$ & 290 & 325 & $\begin{array}{c}328 \\
(313-337)\end{array}$ & 333 & 340 \\
\hline 10) & $\begin{array}{c}106 \\
(105-109)\end{array}$ & 102 & 104 & $\begin{array}{c}42 \\
(35-17)\end{array}$ & 54 & 40 \\
\hline 111 & $\begin{array}{c}156 \\
(152-160)\end{array}$ & 149 & 165 & $\begin{array}{c}171 \\
(168-180)\end{array}$ & 168 & 180 \\
\hline (2) & $\underset{(133-147)}{137}$ & 129 & 11 & $\begin{array}{c}170 \\
\{16 b-17 b\}\end{array}$ & 164 & 482 \\
\hline 13) & $\begin{array}{c}104 \\
(90-121)\end{array}$ & 78 & 117 & $\begin{array}{c}200 \\
(176-219)\end{array}$ & 153 & 215 \\
\hline 14) & $\begin{array}{c}162 \\
(13)-172)\end{array}$ & 137 & 156 & $\begin{array}{c}264 \\
(259-335)\end{array}$ & 254 & 310 \\
\hline (5) & $\begin{array}{c}327 \\
(298-352)\end{array}$ & 327 & 34 & $\begin{array}{c}355 \\
(325-376)\end{array}$ & 380 & 348 \\
\hline (6) & $\begin{array}{c}374 \\
|337-590|\end{array}$ & 379 & 384 & $\begin{array}{c}100 \\
(384-415)\end{array}$ & 446 & 103 \\
\hline$|7|$ & $\begin{array}{c}125 \\
(113-137)\end{array}$ & - & 125 & $\begin{array}{c}143 \\
\{135-156\}\end{array}$ & - & 137 \\
\hline 18: & $\begin{array}{c}35 \\
\{33-37\}\end{array}$ & 34 & 33 & $\begin{array}{c}39 \\
(56-40)\end{array}$ & 36 & 37 \\
\hline (9) & $\begin{array}{c}109 \\
(102-119)\end{array}$ & 100 & 112 & $\begin{array}{c}119 \\
(107-126)\end{array}$ & 116 & 119 \\
\hline 201 & $\begin{array}{c}60 \\
(5 t-62)\end{array}$ & 58 & 60 & $\begin{array}{c}65 \\
(5)-71)\end{array}$ & 61 & 64 \\
\hline 211 & $\begin{array}{c}98 \\
(92-107)\end{array}$ & 91 & 100 & $\begin{array}{c}106 \\
(97-110)\end{array}$ & 104 & 112 \\
\hline 221 & $\begin{array}{c}20 \\
(18-20]\end{array}$ & 20 & 18 & $\begin{array}{c}21 \\
(20-22)\end{array}$ & 20 & 20 \\
\hline $231 *$ & 215 & 215 & 219 & & & \\
\hline $241+$ & 86 & 78 & - & & & \\
\hline A & 74.3 & 77.2 & 74.1 & 74.1 & 83.9 & 75.0 \\
\hline B & 16.4 & 64.6 & 68.8 & 69.1 & 67.4 & 66.5 \\
\hline C & 34.6 & 35.1 & 32.3 & 12.8 & 16.2 & 11.5 \\
\hline 0 & 88.7 & 86. 5 & 88.1 & 99.4 & 97.6 & 95.7 \\
\hline E & 110.0 & $\$ 10.0$ & 112.0 & 112.0 & 111.0 & 106,3 \\
\hline $\mathbf{F}$ & 21.9 & 20.3 & 20.8 & 33.1 & 30.7 & 35.4 \\
\hline
\end{tabular}



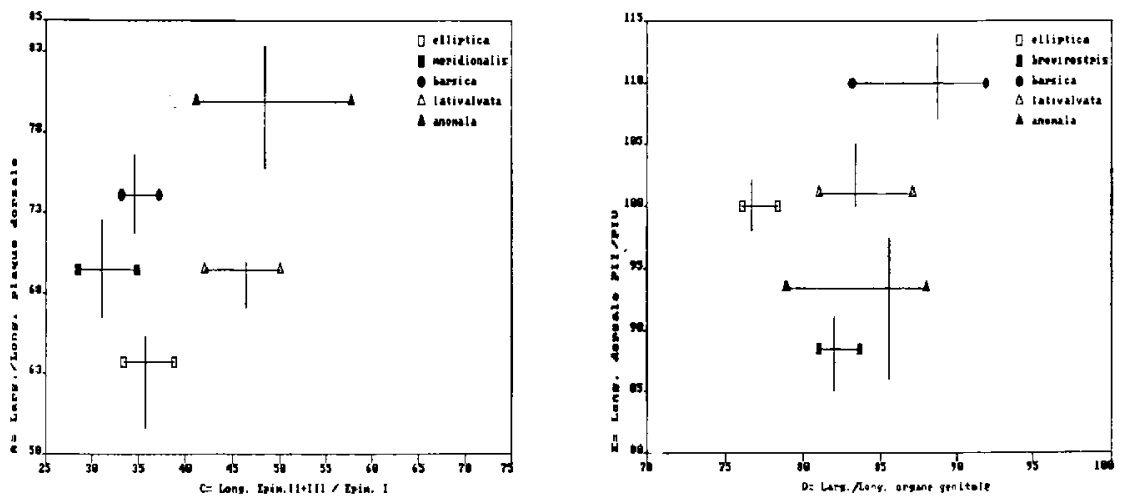

Fig. 4. Taux de variabilité de quelques indices morphologiques.

description de $T$. hispanica concluait « $T$. barsica und $T$. hispanica einander ausserst nahe zu stehen scheinen. $T$. barsica ist aber länglicher und hat im weiblichen Geschlecht eine kürzere gemeinsame Medianhat der Ep. II + III. Weitere Untersuchungen sind erforderlich, um die Artberichtigung dieser und anderer Formen zu klaren. " La comparaison (Tableau VI) avec le matériel typique (prép. $\mathbf{n}^{\circ}$ 2758 et $n^{\circ} 4316$ ), offert par le Musée de Stockholm, a démontré l'uniformité quantitative et qualitative, pour les $\sigma$, et seulement des petites différences pour les $Q$ (longueur plus grande de l'épimère II + III et la morphologie de la face dorsale); ce ne semble pas suffisant, à notre avis, pour distinguer les deux espèces mais n'ayant pas encore de certitudes sur la valeur diagnostique des caractères cités auparavant et, ne disposant pas de données pour mettre en évidence des différences écologiques, nous souhaitons, en suivant Lundblad, que des études ultérieures puissent éclaircir le statut taxonomique de l'espèce. Au contraire $T$. mucronata Viets (1955) décrite sur un matériel recueilli dans l'A pennin ombrien des Marches (prép. $\mathrm{n}^{\circ} 7202$ Musée de Frankfurt) paraît être identique à $T$. barsica. En effet, la superposition de presque tous les caractères des dimensions et des indices morphologiques reportés dans le tableau VI est évidente. Egalement l'examen des caractères qualitatifs nous a convaincus de la synonymie des deux espèces, surtout en prenant en considération que le o décrit par Viets est un exemplaire jeune comme le montre l'absence des empreintes chitineuses sur l'écusson dorsal et le développement réduit de l'aire post-génitale. La longueur du rostre, qui est retenue descriminante par l'auteur allemand, a une variabilité reconnue.

L'examen d'une $O$ ( $n^{\circ} 7203$ ), que Viets a recueillie dans la même localité, a permis de relever en outre la présence des deux glandes anales localisées en dessous du pore excréteur, comme nous l'avons déjà fréquemment observé dans les populations de $T$. barsica que nous avons étudiées. Bien que dans chaque groupe systématique, s'opposent plus ou moins à l'ćtat latent, deux tendances opposées, celle d'unir les espèces et celle de les séparer, nous pensons que $T$. mucronata est synonyme de $T$. bursica.

\section{A. 8. Torrenticola brevirostris (Halbert 1911) (fig. 7 o-r)}

Distribution : Holarctique.

Ecologie : rhéobionte, eurytherme. 
Stations : C2-1 (1 m, $1 \mathrm{f}) ; \mathrm{C} 2-2(2 \mathrm{~m}, 2 \mathrm{f}) ; \mathrm{C} 2-3$ (1 f) ; $\mathrm{C} 2-4(2 \mathrm{~m}, 2 \mathrm{f}) ; \mathrm{C} 2-5(2 \mathrm{~m}) ; \mathrm{C} 2-7(1 \mathrm{~m}$, $2 \mathrm{f})$; C2-10 (2 m, $2 \mathrm{f}, 2 \mathrm{n})$; C2-17 (1 m, $1 \mathrm{f})$; M3-2 $(1 \mathrm{~m})$; M3-4 (1 m, $1 \mathrm{f}, 1 \mathrm{n})$; M3-9 (1 f). Total : 29 individus.

L'espèce, non signalée pour la faune italienne, semble être diffuse dans l'A pennin abruzzais et calabrais à des altitudes comprises entre $0 \mathrm{~m}$ et $1600 \mathrm{~m}$. Comme on peut le déduire des données reportées sur la figure $5, T$. brevirostris supporte de considérables amplitudes thermiques $\left(8-26^{\circ} \mathrm{C}\right)$, bien qu'elle présente un préférendum pour les basses températures.

Les valeurs des caractères ét udiés ont mis en évidence, chez le $\sigma$, une constance dans la longueur des épimères II + III, une zone génitale bien développée et distanciée de la partie terminale du corps (Tableau V, fig. 7 p), un appareil éjaculateur caractérisé par un développement accentué du « relief antérieur ", deux robustes bras proximaux et une cavité proximale peu ample (fig. 7 r). Sont caractéristiques, dans les deux sexes, le second article du palpe (de longueur inférieure au $P$ IV), le pore excréteur et les glandes anales bien distanciées de la ligne de sclérotisation de l'accroissement primaire. Les caractères exposés ci-dessus se retrouvent aussi dans la description de $T$. histriensis (Walter) par Bader (1955). L'étude du matériel typique (exemplaire $\&$ - prép. XXV/81), mis à notre disposition par le Musée de Bâle, ne nous a pas permis đe remarquer des différences quantitatives et qualitatives avec $T$. brevirostris; par conséquent nous pensons opportun de mettre en synonymie les deux espèces. Bader, dans le même travail, affirmait qu'on devait considérer que $T$. histriensis était très proche de $T$. turkestanica Sokolov 1926 et $T$. sandalensis Sokolov 1926. L'impossibilité d'observer le matériel typique et les descriptions incomplètes de l'auteur russe, même s'ils ne nous permettent pas d'affirmer leur validité spécifique, n'empêchent pas d'avoir quelques doutes sur le statut taxonomique des deux espèces, comme l'a déjà observé Szalay (1933 b) pour la synonymie entre $T$. brevirostris et $T$. sandalensis. Outre cela, l'examen des exemplaires jeunes de $T$. brevirostris a permis de mettre en évidence les caractéristiques morphologiques identiques à celles attribuées à T. maglioi (Koenike 1908) signalée d'Italie et de nombreuses localités d'Europe centrale. De telles observations ne nous permettent pas d'être des défenseurs convaincus de la valeur spécifique de $T$. maglioi.

\section{B. Sous-genre Monatractides Viets 1926}

B. 1. Torrenticola stadleri (Waiter 1924) (fig. 8 a-d)

Distribution : Italie, Corse, Espagne, France, Allemagne, Tchécoslovaquie, Roumanie, Hongrie, Yougoslavie, Grèce.

Ecologie : eurytherme.

Stations : M2-1 (1 f) ; M3-3 (1 m, $3 \mathrm{f}, 1 \mathrm{n})$; M3-4 (4 m, $9 f, 4 n)$; M3-5 (2 m, 6f, $2 \mathrm{n})$; M3-6 (1 m, $1 \mathrm{f})$; M3-7 (1 m, $1 \mathrm{f})$; M3-9 (1 f ) ; M3-10 (2f, $1 \mathrm{n})$. Total : 41 individus.

L'espèce est signalée de façon douteuse en Italie par Maglio (1956) sur un exemplaire $\odot$ recueilli dans le fleuve Esino (Apennin ombrien et des Marches). Les exemplaires étudiés proviennent seulement de l'Apennin calabrais-lucanien où l'espèce est en majeure partie répartie à des altitudes $<300 \mathrm{~m}$ et jusqu'à $800 \mathrm{~m}$ (fig. 5). Les milieux sont caractérisés par une température comprise entre $13^{\circ}$ et $29^{\circ} \mathrm{C}$ et par une vitesse du courant réduite. Peut-être c'est l'espèce la moins réophile du genre (Angelier 1953).

$T$. stadleri semble être bien caractérisée morphologiquement, l'appareil éjaculateur, en particulier, est d'une dimension considérable avec une cavité proximale peu développée (fig. $8 \mathrm{~d}$ ). Le tableau VII se réfère aux valeurs des caractères d'un exemplaire $\sigma$.

\section{B, 2. Torrenticola madritensis (Viets 1930) (fig. $8 \mathrm{e}-\mathrm{g}$ )}

Distribution : Italie, Espagne, France, Suisse, Yougoslavie, Angleterre, Autriche.

Ecologie : eurytherme, interstitiel.

Stations : M3-9 (3 f) ; M3-15 (1 m, $1 \mathrm{f})$. Total : 5 individus.

L'espèce a été signalée pour l'Italie dans la faune interstitielle du torrent S. Bernardino-Lago Maggiore (Schwoerbel 1961). Les exemplaires recueillis proviennent de l'Apennin calabrais dans deux localités situées à 450 et $1450 \mathrm{~m}$ d'altitude. Selon Schwoerbel (1962) elle fait partie du groupe d'espèces qui vivent dans les milieux superficiels de la zone méditerranéenne et dans les eaux interstitielles des Alpes. Sur le peu de données de la littérature, il n'est pas exclu que l'espèce puisse être retrouvée dans le 

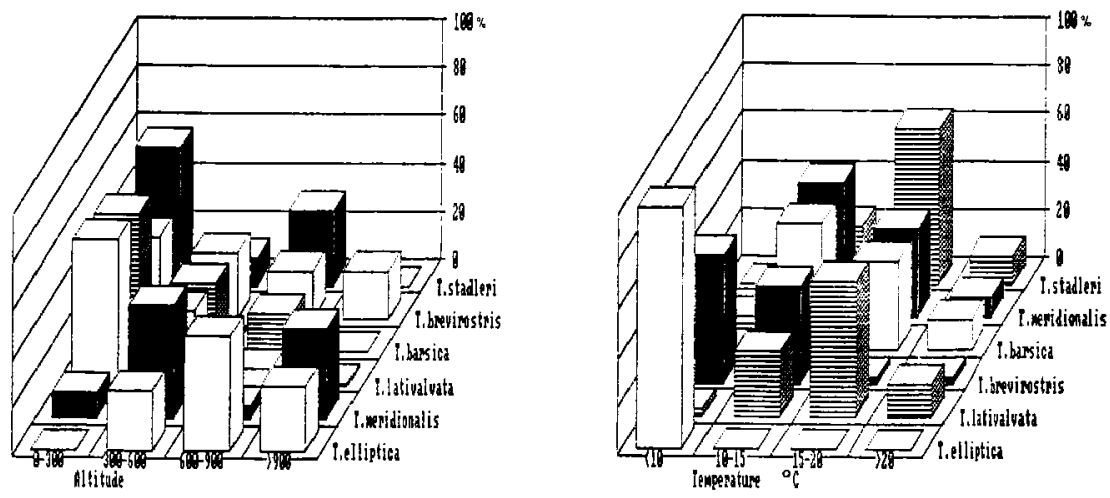

Fig. 5. Répartition ( $(\%)$ des espèces en fonction de l'altitude et de la température.

bassin méditerranéen, soit dans le milieu interstitiel où elle migrerait par suite de variations saisonnières thermique et hydraulique des rivières, soit dans les eaux superficielles. Cette dernière condition ne pourrait se réaliser dans les cours d'eau alpins et d'Europe centrale où le développement d'une faune riche et bien diversifiée ne permettrait pas à l'espece hyporhéique d'émigrer en surface, à cause des phénomènes de compétition (Angelier 1952, 1957). Dans la figure 8 sont reportées quelques caractéristiques morphologiques de l'espèce faciles à observer et dans le tableau VII les dimensions des caractères relatifs à un exemplaire ${ }^{\circ}$.

\section{Sous-genre Rusetria Thor}

C. 1. Torrenticola ungeri (Szalay 1927) (Fig. 8 h-m)

Distribution : Corse, Espagne, France, Autriche, Pologne, Hongrie, Tchécoslovaquie, Yougoslavie, Turquie.

Ecologie : eurytherme, interstitiel.

Stations : M3-4 (1 m, 1 f, $1 \mathrm{n})$; M3-9 (1 m, $3 \mathrm{f})$; M3-15 (1 m, $4 \mathrm{f}$ ). Total : 7 individus.

Signalé pour la première fois en Italie. On l'a trouvé dans les eaux superficielles de l'Apennin calabrais dans des stations situées entre 50 et $1350 \mathrm{~m}$ d'altitude. Dans la littérature les indications les plus nombreuses se reportent au milieu interstitiel comme en témoigne l'intégration de l'espèce dans la "Stygofauna mundi » (Schwoerbel 1986). Pour ces raisons, l'hypothèse concernant la distribution géographique de $T$. madritensis, peut être valable aussi pour cette espèce. Le matériel n'a permis de mesurer qu'un exemplaire $O^{*}$ (Tableau VII).

\section{2. Torrenticola amplexa (Koenike 1908) (fig. $8 \mathrm{~m}-\mathrm{p}$ )}

Distribution : Europe, Japon.

Ecologie : eurytherme, limicole.

Stations : C2-15 (2 f) ; M3-4 (5 f, $2 \mathrm{~m}$ ). Total : 9 individus.

Espèce signalée également pour la permière fois en Italie. Les exemplaires proviennent de deux stations de l'Apennin méridional situées à basse altitude.

D'après la littérat ure elle semble typique des petits ruisseaux où elle vit dans le sédiment (Viets 1957), indifférente à la vitesse du courant et aux variations thermiques. Elle a été retrouvée dans un grand nombre de ruisseaux de piémont de Forêt Noire (200-500 m) caractérisés par un courant faible et par de fortes amplitudes thermiques (Schwoerbel 1959). Dans le tableau VII nous reportons les dimensions relatives au $0^{\circ}$. 
Tableau VII. Mesures des caractères de quatre exemplaires mâles de $T$. madritensis, $T$. stadleri, $T$, ungeri et T. amplera.

\begin{tabular}{|c|c|c|c|c|}
\hline & & mess & & \\
\hline car. & staderi & edritensis & unger i & $\begin{array}{l}\text { applexi } \\
\text { prep. }\end{array}$ \\
\hline 1 & 825 & 604 & 598 & $\$ 70$ \\
\hline 2 & 710 & 494 & 475 & 427 \\
\hline 3 & 1000 & 636 & 731 & 665 \\
\hline 1 & 780 & 627 & 541 & 541 \\
\hline 5 & 240 & 196 & - & - \\
\hline 6 & 190 & 117 & 141 & 125 \\
\hline 7 & 223 & 156 & 153 & 137 \\
\hline 8 & 52 & 58 & 98 & - \\
\hline 9 & 390 & 259 & 299 & 262 \\
\hline 10 & 98 & 117 & 78 & 94 \\
\hline 11 & 219 & 157 & 149 & 153 \\
\hline 12 & 197 & 118 & 129 & 153 \\
\hline 13 & 215 & 194 & 149 & 117 \\
\hline 14 & 370 & 202 & 211 & 157 \\
\hline 15 & 235 & 171 & 352 & 294 \\
\hline 16 & 290 & 195 & 390 & 341 \\
\hline 17 & * & 80 & 125 & 117 \\
\hline 18 & 30 & 26 & 12 & J5 \\
\hline 19 & 85 & 62 & 112 & 91 \\
\hline 20 & 57 & 58 & 64 & 57 \\
\hline 21 & 71 & 62 & 100 & 95 \\
\hline 22 & 29 & 32 & 20 & 17 \\
\hline 23 & 345 & - & 203 & - \\
\hline 24 & 117 & - & 63 & - \\
\hline
\end{tabular}

\section{Conclusions}

Malgré le caractère préliminaire de l'étude, il est possible de mettre en évidence quelques résultats faunistiques, taxonomiques, zoogéographiques et écologiques intéressants.

Cinq espèces sont nouvelles pour la faune italienne. $T$. mucronata a été mise en synonymie avec $T$. barsica. Sont ainsi connues pour l'Italie 13 espèces du genre Torrenticola, même si $T$. maglioi suscite quelque doute pour sa ressemblance morphologique aux individus peu développés de T. brevirostris.

L'étude biométrique conduite sur de nombreux caractères fait apparaît re plusieurs données significatives afin d'individualiser quelque valeur descriminante au niveau spécifique. Malgré les difficultés rencontrées, il est possible d'affirmer que, chez les $\sigma$, cinq indices morphologiques possèdent une valeur discriminante certaine tandis que la plupart des caractères dimensionnels n'ont aucun poids dans la diagnose à cause de leur grande variabilité due, en particulier, à l'accroissement du type allométrique. Le tableau VIII, qui réunit les indices morphologiques plus informatifs et calculés sur des individus bien développés, a mis en évidence que :

1) le rapport largeur/longueur de la face dorsale (index A) présente, chez les or, une faible valeur pour T. elliptica et $T$. similis $(64 \%)$, pour $T$. meridionalis et $T$. lativalvata $(69 \%)$, et une valeur plus élevée pour les formes peu arrondies $T$. brevirostris et $T$. anomala. Une situation assez semblable se retrouve chez les $Q$.

2) L'index $C$ (rapport entre la longueur des épimères II + III / longueur épimères I), montre peu de variabilité intraspécifique, individualisant chez les $\sigma$, deux groupes d'espèces ( $T$. meridionalis, $T$. elliptica, $T$. similis, $T$. barsica - vs - T. brevirostris, $T$. lativalvata, $T$. anomala, $T$. crenobia). Chez les $Q$ cet indice réussit seulement à séparer $T$. brevirostris et $T$. anomala $(6,2$ vs $23,4 \%)$;

3) La forme de l'organe génital (indice D) semble utile, surtout chez les $\sigma$, pour caractériser la plus grande partie des espèces examinées ;

4) Le rapport P2/P4 (indice E) permet d'indivualiser, dans les deux sexes, les espèces avec des valeurs inférieures à 100 ( $T$. brevirostris et $T$. anomala), à peu près égales à 100 ( $T$. elliptica, $T$. meridionalis, $T$. lativalvata, $T$. crenobia) et supérieures à $100(T$. barsica) ; 
Tableau VIII. Indices morphologiques des mâles et femelles des espèces du sous-genre Torrenticola s. str.

\begin{tabular}{|c|c|c|c|c|c|c|c|c|}
\hline \multicolumn{9}{|c|}{ MAESS } \\
\hline indices & elliptica & $\begin{array}{l}\text { sisilis } \\
\text { prep. } 290\end{array}$ & ser idionalis & barsica & brevirastris & Lativalvate & Inomala & $\begin{array}{l}\text { erenotsia } \\
\text { arep.310 }\end{array}$ \\
\hline$\ddot{A}$ & 63.7 & 63.0 & 69.5 & 74.3 & 77.0 & 69.5 & 79.6 & 72.6 \\
\hline$B$ & 61.9 & 67.5 & 64.8 & 76.4 & 75.5 & 61.1 & 66.7 & 58.3 \\
\hline 6 & 35.7 & 35.1 & 31.0 & 34.6 & 37.8 & 46.4 & 48.1 & 53.2 \\
\hline 0 & 76.7 & 73.7 & 80.7 & 68.7 & 02.0 & 83.3 & 85. 6 & 77.5 \\
\hline$E$ & 100.0 & 103.0 & 100.0 & $\$ 10.0$ & 68.1 & 101.0 & 93.4 & 102.7 \\
\hline$F$ & 26.8 & 29.6 & 27.1 & 21.9 & 30.1 & 19.5 & 22.0 & 21.0 \\
\hline
\end{tabular}

FEMELLES

\begin{tabular}{|c|c|c|c|c|c|c|c|c|}
\hline indices & elliptica & $\begin{array}{l}\text { sibilis } \\
\text { prep. 29! }\end{array}$ & eer idional is & barsica & brevirostris & Lativaluata & anosald & $\begin{array}{l}\text { crenobia } \\
\text { prep. } 311\end{array}$ \\
\hline $\bar{A}$ & 64.4 & 64.7 & 74.5 & 74.1 & 79.0 & b7.2 & 78,3 & 67,8 \\
\hline B & 65.9 & 64.8 & b6. 1 & 69.1 & 77.5 & 63.3 & 06.1 & 71.6 \\
\hline [ & 13.7 & 12.9 & 10.2 & 12.8 & 8.2 & 11.8 & 23.4 & 10.7 \\
\hline 0 & 87.0 & 92.6 & 93.3 & 99.4 & 94.4 & 94.7 & 94.5 & 90.9 \\
\hline E & 102.0 & 106.0 & 102.0 & 112.0 & 89.3 & 104.3 & 88.9 & 107.7 \\
\hline$F$ & 34.5 & 30.1 & 34.0 & 33.1 & 37.4 & 34.6 & 34.9 & 36.4 \\
\hline
\end{tabular}

5) Le rapport entre la distance organe génitalpartie terminale du corps sur la longueur ventrale (indice F) caractérise, chez les $\sigma^{*}$, deux groupes d'espèces $T$. elliptica, $T$. similis, $T$. meridionalis, $T$. brevirostris, qui présentent un organe génital déplacé antérieurement, par rapport à $T$. lativalvata, $T$, anomala, $T$. crenobia et $T$. barsica.

De telles informations, intégrées avec celles déduites de l'étude du matériel typique, ont permis d'établir 4 nouvelles synonymies ( $T$. longirostris, $T$. piriformis, $T$. mucronata et $T$. histriensis), de mettre en doute le statut taxonomique de deux entités ( $T$. maglioi et $T$. hispanica) et de retenir $T$. microphallus comme une espèce valable.

L'approche suivie a donné l'opportunité de redessiner (pour les $\sigma$ ) ces principaux caractères, pas toujours suffisamment décrits dans la littérature. Il nous a semblé particulièrement utile de proposer, autant que possible, les illustrations de l'appareil éjaculateur non décrites par plusieurs auteurs (Lundblad 1972, Barr 1972, 1979, Gerecke 1990), caractère dont l'examen attentif peut être extrêmement utile pour l'identification de plusieurs taxa.

Toutes les données déjà énoncées ont été utilisées pour proposer la clef analytique donnée ici.

Avec les réserves déjà mentionnées, il est possible de faire quelques considérations zoogéographiques.

D'après les données relatives à l'Apennin abruzzais et calabrais, les deux secteurs où sont situées la majorité des stations, on peut déduire clairement une différente distribution des espèces (Tableau IX et fig. 3). Dans le premier l'espèce la plus fréquente est $T$. brevirostris, suivie de $T$. elliptica, qui est l'espèce la plus abondante ; dans le secteur calabrais, au contraire, l'espèce la plus fréquemment recueillie a été $T$. meridionalis suivie de $T$. lativalvara, qui représente $42 \%$ des récoltes. Le nombre total des espèces est plus grand dans l'Apennin calabrais (10 vs 6), suivant des données présentes en littérature 
Tableau IX. Distribution des espèces dans les stations étudiées.

Légende : Al $=T$. anomala $;$ A2 $=T$. crenobia $;$ A3 $=T$. elliptica $;$ A4 $=T$. similis: $\mathrm{AS}=T$. meridionalis; $\mathbf{A 6}=T$. lativalvata; $\mathbf{A} 7=T$. barsica; $\mathbf{A} 8=T$. brevirostris; $\mathrm{B1}=T$. stadleri; $\mathrm{B2}=T$. madritensis $; \mathrm{C} 1=T$. ungeri $; \mathrm{C2}=L$. amplexa .

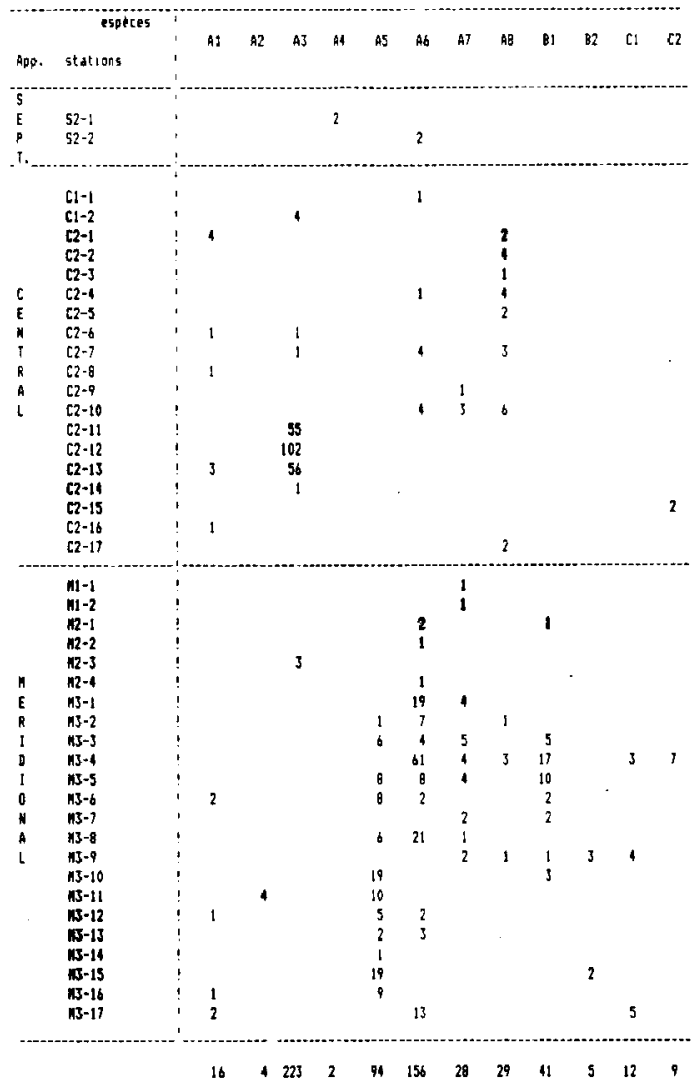



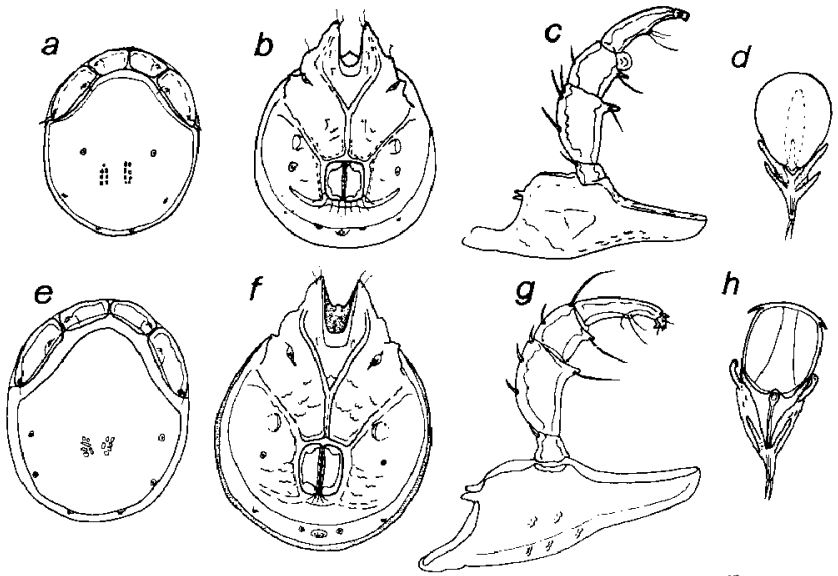

h
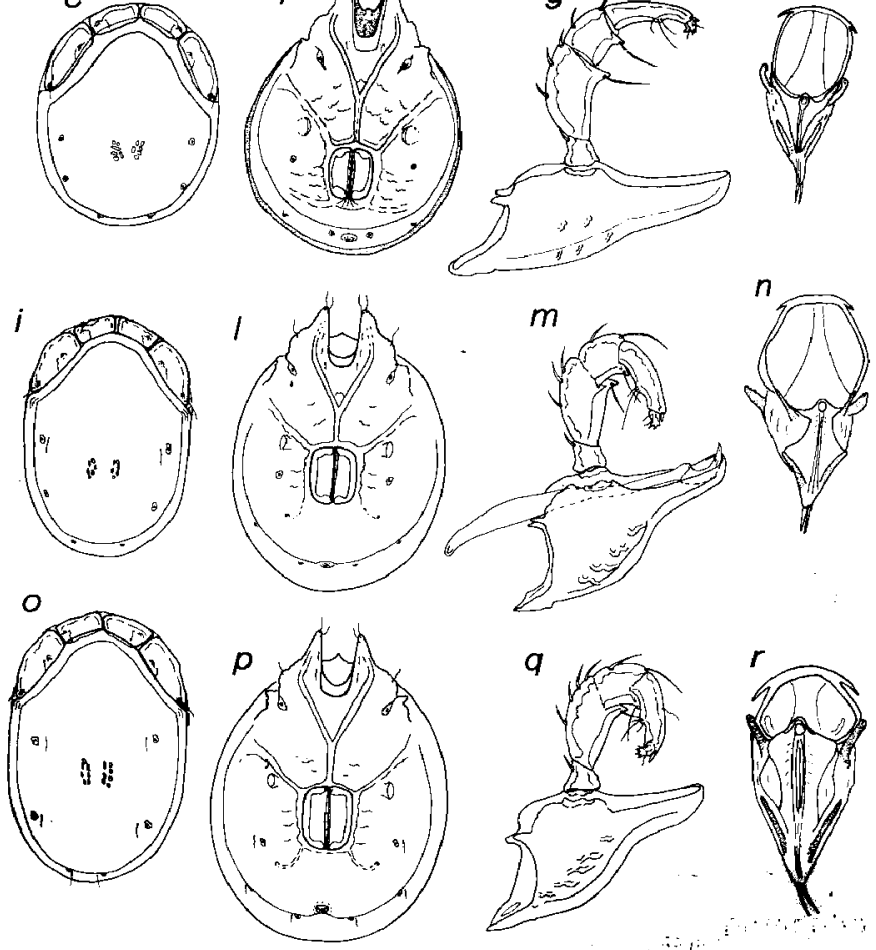

abefilop

$0.50 \mathrm{~mm}$
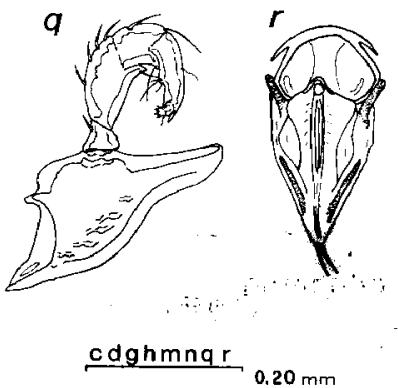

Fig. 6. $T$. anomala : a-d ; $T$. crenobia : e-h ; T. elliptica : $\mathrm{i}-\mathrm{n} ; T$, similis : $0-\mathrm{r}$. $(a, e, i . o=$ face dorsale $; b, f, l, p=$ face ventrale $; c, g, m, q=$ gnathosome $; d, h, p, r=$ appareil éjaculateur). 

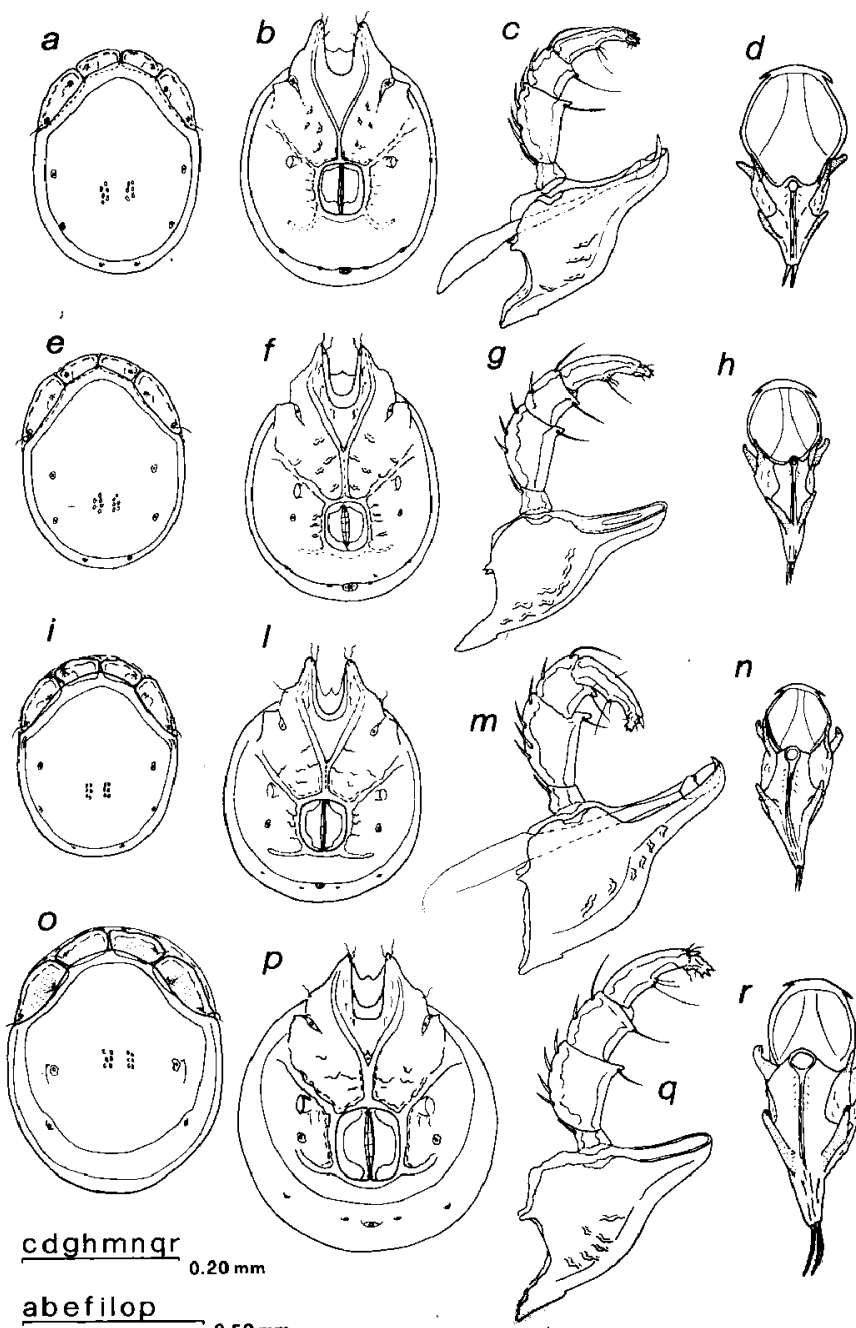

abefilop $0.50 \mathrm{~mm}$

Fig. 7. T. meridionalis : a-d ; T. lativalvata : e-h ; T. barsica : i-n ; T. brevirostris : o-r. $(a, e, i, o=$ face dorsale; b. $f, l, p=$ face ventrale; $c, g, m, q=$ gnathosome $; d, b, p, r=$ appareil éjaculateur $)$. 

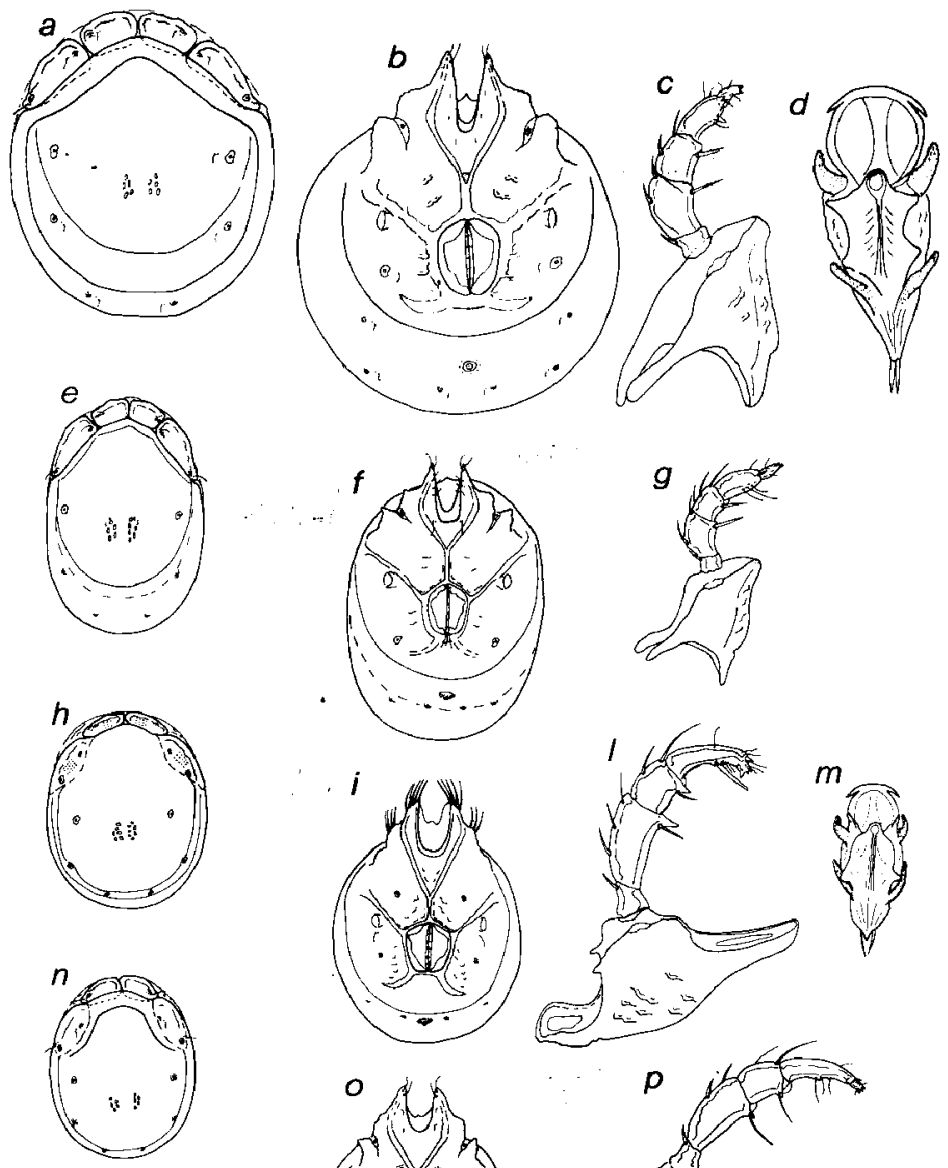

$\stackrel{c d g l m p}{c} 0.20 \mathrm{~mm}$
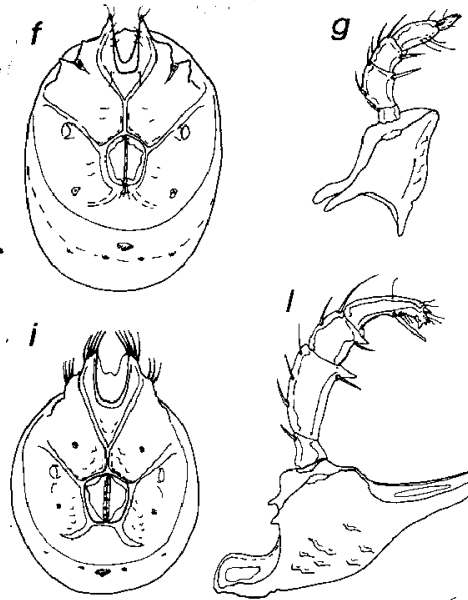

abef hino

$0.50 \mathrm{~mm}$
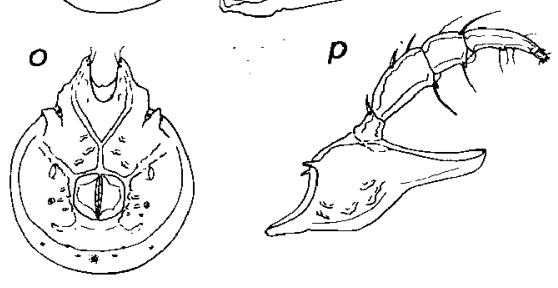

Fig. 8. T. stadleri : a-d ; $T$. madritensis : e-g ; $T$. ungeri $: \mathrm{h}-\mathrm{m} ; T$. amplexa : n-p.

(a, e, h. $\mathrm{n}=$ face dorsale $; \mathrm{b}, \mathrm{f}, \mathrm{i}, \mathrm{o}=$ face ventrale $; \mathrm{c}, \mathrm{g}, \mathrm{l}, \mathrm{p}=$ gnathosome $; \mathrm{d}, \mathrm{m}=$ appareil éjaculateur). 
qui mettent en évidence un appauvrissement faunistique dans le sens Sud-Nord pour le genre Torrenticola, considérée comme « relicte de la faune chaude du tertiaire " (Angelier 1952, 1957). Quatre espèces ( $T$. meridionalis, $T$. stadleri, $T$. ungeri, $T$. madritensis) ont été recueillies seulement dans l'Apennin méridional ; $T$. lativalvata, signalée aussi en Afrique du Nord et en Calabre, a réussi à pénétrer dans lc secteur le plus septentrional de l'Apennin (Abetone) ; $T$. similis au contraire, semble avoir une distribution plus septentrionale, n'étant encore pas trouvée dans l'Apennin central et méridional. Des considérations plus précises et définitives ne sont pas faciles pour ce taxon insuffisamment étudié dans le bassin de la Méditerranée.

Pour la Méditerranée, le genre a été étudié dans la Péninsule Ibérique, en Corse, en France et, occasionnellement, en Afrique du Nord et Yougoslavie (cf. Viets 1956, Viets 1957). Les résultats repris par nous, outre qu'ils ont comblé en partie cette lacune, ont permis de mettre en évidence l'importance du microhabitat, qui est déterminé par la typologie du cours d'eau, pour expliquer le nombre et la distribution géographique des espèces.

L'entité géomorphologique de l'Apennin abruzzais et calabrais détermine, dans les deux cas, des cours d'eau brefs mais avec des fluctuations de régimes. Dans l'Apennin abruzzais un régime du type «Apennin central » est caractérisé par des débits minima pendant l'été et maxima pendant le printemps et/ou l'automne. La variation des débits est en général peu accentuée à cause de la présence des roches calcaires perméables qui régularisent les écoulements. Dans l'Apennin calabrais le régime est dans la plupart des cas de type torrentiel, avec de tres fortes et imprévisibles crues qui déterminent dans la partie inférieure, de larges lits détritiques dérivés de l'érosion des reliefs cristallins imperméables, comme cela a déjà été observé dans d'autres zones de la Méditerranée (Angelier 1959, 1961, Gerecke 1990), Durant l'été, les débits faibles ou même nuls ne permettent pas d'occuper le lit entier du fleuve et la vie aquatique est alors influencée par l'augmentation de la température et de la salinité, la diminution de l'oxygène dissous et par le type de substrat. La continuité spatiale des microécosystèmes durant certaines périodes saisonnières, pourrait être maintenue, dans des milieux torrentueux, en fonction de la capacité d'effectuer des migrations dans un milieu pétricole-hyporrhéique connu pour la plupart des représentants du genre (Angelier et al. 1985). Seul le déterminisme écologique pourrait expliquer la diversification des espèces du genre Torrenticola dans l'aire de la Méditerranée et, donc, I'appauvrissement faunistique Sud-Nord (précédemment indiqué), mis en évidence également dans le secteur des Apennins.

Seules des études ultérieures dans d'autres localités de la Méditerranée (Alpes-Maritimes, Sicile, Sardaigne) qui sont en cours de réalisation, pourront confirmer les hypothèses formulées et expliquer la relation existante entre la distribution géographique et la distribution de la niche écologique correspondante.

Clé des espèces italiennes du sous-genre Torrenticola

1 Infracapitulum ventralement presque droit (fig. 6 d) ............. 2

- Infracapitulum ventralement recourbé (fig. (n) ............... 3

2 Deuxième article du palpe (P2) plus long que le $\mathrm{P3}$, chambre proximale de l'appareil éjaculateur dépourvue des appendices sclérifiés (proximal horns) sur le bord supérieur (fig. $6 \mathrm{~d}$ ), plaque génitale de petites dimensions

T. anomala

- P2 de dimensions inférieures au P4, appareil éjaculateur avec appendices sclérifiés $(f i g .6 \mathrm{~h}$ ), plaque génitale plus grande

T. crenobia

3 Infracapitulum avec un rostre bien développé, pore excréteur situé à la limite de la ligne de sclérification primaire................... - Infracapitulum avec un rostre peu développé, pore excréteur bien distancé de la ligne de sclérification primaire

T. brevirostris

4 Longueur des épimères II + III sur les épimères $I($ index $\mathrm{C})<40 \% \ldots \ldots 5$

- Index $C>40 \% \ldots \ldots \ldots \ldots . .$. . lativalvata

5 P2 de dimensions comme le P4 ; largeur/longueur de l'écusson dorsal (index A) $<70 \%$; area post-génitale bien développée (index $F$, voir texte) 6

- P2 plus long que P4; index $\mathrm{A}>$ $70 \% ;$ area post-génitale réduite ... T. barsica 
6 forme du corps allongée (index $\mathrm{A}<$ (64 \%) ; organe genital bien développé 7

- forme du corps allongée (index $\mathrm{A}>$ $67 \%$ ), organe génital plus petit ...

T. meridionalis

7 Appareil éjaculateur avec une chambre proximale très ample et les sclérites proximaux déplacés postérieurement (fig. 6 n) ; pore excréteur et glandes anales sur la ligne de sclérotisation primaire - appareil éjaculateur avec une chambre proximale réduite, sclérites proximaux déplacés plus antérieurement $($ fig. $6 \mathrm{r}$ ) ; pore excréteur dans une dépression de la sclérotine primaire ............
Cicolani (B.) \& Sisino (L.). 1983. - Idracnnidi del fiume Vetoio (Acarina, Prostigmata, Hydrachnellae). (2e Contributo alla conoscenza delle Idracnelle, dell'Appennino). Riv. Idrobbiol., $22(2): 89-99$.

Cicolani (B.), Bianchi Bullini (A.P.) \& Bullini (L.). 1981. -- Morphological and genetic differentiation between Macrocheles glaber and Macrocheles perglaber (Acarina, Mesostigmata). Int. Journ. Acarol., $7: 221-224$.

Di Sabatino (A.) \& Cicolani (B.). 1989. - Torrenticola crenobia n. sp. (Acari, Actinedidae, Torrenticolidae) from a spring of the calabrian Apennines, South Italy. Riv. Idrobiol. (sous presse).

Di Sabatino (A.) \& Cicolani (B.). 1990. - Torrenticola meridionalis n.sp., a new species of water mites (Acari, Actinedida, Torrenticolidae) from the calabrian Apennines (South Italy). Louterbornia, $5:$ :33-48.

Gerecke (R.). 1986. - Le acque interne di Sicilia e la loro fauna : un patrimonio naturale da salvare. Animalia, $13(1 / 3): 217-245$.

Gerecke (R.). 1990. - Sistematische, faunistische und okologische Untersuchungen an Wassermilben aus Sizilien, unter Berücksichtigung mehrerer anderer Invertebratengruppen. Thèse de doctorat, Univ. Freiburg : 310 p. (pas publiee).

Laska (F.). 1953. - Einige neue Wassermilben aus dem Flussgebiete Orava in der Slowakei. Acto Acad. Scient. Nat. MoravoSilesiaca, 25, $9:$ 281-293.

Laska (F.). 1955. - Über einige seltene Wässermilben (Hydrachnellae) aus den zuflüssen des oberen Becva-Flusses in Mähren. Publ. de la Faculté de Sc. Univ. Masaryk, n³64, 219-230.

Lundblad (C.D.). 1942. - Die Arthropodenfauna von Madeira nach den Ergebnissen der Reise von Prof. Dr O. Lundblad Julj-August 1935. 31. Hydrachnellae. Ark. Zool, 34, (5) : 1-122.

Lundblad (C.D.). 1956. - Zur kenntnis süd - und mitteleuropaischer Hydrachnellen. Ark. Zool., 10 (1) : 1-306.

Maglio (C.). 1905. - Secondo elenco d'Idracne del Pavese. Rend. Ist. Lombard. Sci. Lett. (s.2), 38 : 147-154.

Maglio (C.). 1907. - Elenco critico degli Idracnidi italiatii. Rend. 1st. Lombard. Sci. Lett. (s.2), $40: 953.974$.

Maglio (C.). 1909. - Idracarini del Trentino. (Contributo alla conoscenza dell'idracarofauna alpina). Atti soc. ital. Sci natur., Povia, $48: 251-296$.

Maglio (C.). 1956. - Idracnelle dell'Appennino UmbroMarchigiano. Atti Soc, ital. Nat, Povia, $95: 3-4$.

Marucci (V.). 1906. - Contributo alla conoscenza degli idracnidi del I azio. Boll. Soc. Zool. Hal. Anno $7: 282-288$.

Monti (R.). 1910. - Contributo alla biologia degli idracnidi alpini in relazione all'ambiente. Atti. Soc. ital. Sci. nat., Pavia, 49 : 167-243.

Schwoerbel (J.). 1959. - Okologische und tiergeographische Untersuchungen über die Milben (Acari, Hydrachnellae) der Quellen und bâche des südlichen Schwarzwaldes und seiner Randgebiete, Arch. Hydrobiol., (Suppl.) 24, 3 (3-4) : 385-546.

Schwoerbel (J.). 1961. - Hungarohydracanis subterraneus italicus, nov. ssp., die erste Süßwassermilbe (Hydrachnellae) aus dern hyporeischen Grundwasser Itatiens. Mem. Ist. Ltol. Idrobiol., $13: 115-124$

Schwoerbel (J.). 1\%2. - Subterrane Wassermilben (Hydrachnellae und Thrombiidac) aus den Alpen. Zool. Anz, 168 (7-10) : 292-300.

Schwoerbel (J.). 1986. - Acari : " Hydrachnellae ". In Botosaneanu (L.), ed. : Siygofauna Mundi : 652-697.

Sokolow (I.). 1926. - Zwei neue Hydracarinenarten aus der Gattung Alractides C.L. Kock. Zool. Anz. : 72-76. 
Szalay (L.). 1933 a. - Eine neue Hydracarine aus der Gattung Atractides C. L. Koch und das Weibchen von Airactides (R.) ungeri Szalay. Zool. Anz., 104 (7-8) : 201-205.

Szalay (L.). 1933 b. - Über zwei Wassermilben aus der Gattung Atractides C.L. Koch. Zool. Anz., 102 (9-10): 227-236.

Viets (K.). 1955. - Kleine Sammlungen europäischer und außereuropäischer Wassermitben (Hydrachnellae, Acari). Abh. naturw, Ver. Bremen, 34 (1): 1-26.

Viets (K.). 1956. - Die Milben des Süßwassers und des Meeres. Hydrachnelloe und Halacaridae (Acari). II' und III' teil : Katolog und Nomenklator, Fischer Verlag, Jena : 870 p.
Viets (K.O.). 1957. - Wassermilben aus der Salmoniden-Region von Harzbächen, Abh. Noturwiss, Ver. Bremen, 35 (1): 135-161.

Viets (K.O.). 1967. -- Hydracarina. In : Illies (J., ed.) : Limnofauna Europaea, 124-148.

Viets (K.O.). 1987. - Die Milben des Süßwassers (Hydrachnellae und Halacaridae [part.], Acari). Sonderbinde des Naturwiss, Vereins Hamburg, 8 : 1-1012. 